\title{
PRODUCTS OF FAREY GRAPHS ARE TOTALLY GEODESIC IN THE PANTS GRAPH
}

\author{
SAMUEL J. TAYLOR AND ALEXANDER ZUPAN
}

\begin{abstract}
We show that for a surface $\Sigma$, the subgraph of the pants graph determined by fixing a collection of curves that cut $\Sigma$ into pairs of pants, once-punctured tori, and four-times-punctured spheres is totally geodesic. The main theorem resolves a special case of a conjecture made in [APS08] and has the implication that an embedded product of Farey graphs in any pants graph is totally geodesic. In addition, we show that a pants graph contains a convex $n$-flat if and only if it contains an $n$-quasiflat.
\end{abstract}

\section{INTRODUCTION}

The pants graph $\mathscr{P}(\Sigma)$ has emerged as an central object in lowdimensional geometry and topology over the past decade. The most prominent example of the pants graph's importance is the celebrated result of Brock that the pants graph $\mathscr{P}(\Sigma)$ is quasi-isometric to the Teichmüller space $\mathscr{T}(\Sigma)$ with its Weil-Petersson (WP) metric [Bro03]. As a consequence, the large-scale geometry of $\mathscr{P}(\Sigma)$ is the same as that of $\mathscr{T}(\Sigma)$, and $\mathscr{P}(\Sigma)$ may be used to investigate the geometry of Teichmüller space [BMM11, BM07]. As evidence of the further significance of the pants graph, Brock has shown that distances in $\mathscr{P}(\Sigma)$ are coarsely related to volumes of convex cores of quasi-Fuchsian 3-manifolds [Bro03] and volumes of fibered hyperbolic 3manifolds [Bro01]. The pants graph has also been used to study the topology of 3-manifolds. Johnson has developed 3-manifold invariants based on the pants graph of a Heegaard surface [Joh06], and the second author has produced similar results for knots in 3-manifolds [Zup13].

While the above results demonstrate some of the striking connections between the pants graph and low-dimensional manifolds, the intrinsic geometry of the pants graph remains relatively unexplored. Observing that the mapping class group of $\Sigma$ acts naturally on $\mathscr{P}(\Sigma)$, we note one important theorem about the rigidity of $\mathscr{P}(\Sigma)$, which has been proved by Margalit:

The first author is partially supported by NSF RTG grants DMS-0636557 and DMS1148490. The second author is supported by the National Science Foundation under Award No. DMS-1203988. 
Theorem 1.1. [Mar04] Any automorphism of $\mathscr{P}(\Sigma)$ is induced by an element of the mapping class group of $\Sigma$.

A similar result has been obtained by Aramayona with regard to subsurfaces and inclusion maps. We define a multicurve $Q$ to be a collection of pairwise disjoint simple closed curves in $\Sigma$, and we let $\mathscr{P}_{Q}(\Sigma)$ be the the full subgraph of $\mathscr{P}(\Sigma)$ consisting of all pants decompositions containing $Q$. Aramayona has proved

Theorem 1.2. [Ara10] Suppose $\Sigma^{\prime}$ and $\Sigma$ are surfaces, and $i: \mathscr{P}\left(\Sigma^{\prime}\right) \rightarrow$ $\mathscr{P}(\Sigma)$ is an injective simplicial map. Then there exists a multicurve $Q$ in $\Sigma$ such that $i\left(\mathscr{P}\left(\Sigma^{\prime}\right)\right)=\mathscr{P}_{Q}(\Sigma)$.

Thus, in addition to being rigid with respect to automorphisms, the pants graph is rigid with respect to inclusions. A natural problem which follows is to determine the geometry of the subspaces $\mathscr{P}_{Q}(\Sigma)$ within $\mathscr{P}(\Sigma)$. Although the correspondence between $\mathscr{P}(\Sigma)$ and $\mathscr{T}(\Sigma)$ mentioned above is a quasi-isometry, we may look to $\mathscr{T}(\Sigma)$ for clues as to how these subspaces might behave. By a result of Masur, the boundary of the WP metric completion $\overline{\mathscr{T}}(\Sigma)$ of $\mathscr{T}(\Sigma)$ consists of strata of the form $\mathscr{T}\left(\Sigma^{\prime}\right)$, where $\Sigma^{\prime}$ is the noded Riemann surface obtained by pinching each curve in a multicurve $Q$ [Mas76]. Moreover, each stratum is totally geodesic in $\overline{\mathscr{T}}(\Sigma)$ : every geodesic in $\bar{T}(\Sigma)$ with endpoints in $\mathscr{T}\left(\Sigma^{\prime}\right)$ is contained entirely in $\mathscr{T}\left(\Sigma^{\prime}\right)$ [Wol03].

By interpreting these results in terms of $\mathscr{P}(\Sigma)$, Aramayona, Parlier, and Shackleton have made the following conjecture:

Conjecture 1. APS08] Suppose that $\Sigma^{\prime}$ and $\Sigma$ are surfaces, and $i: \mathscr{P}\left(\Sigma^{\prime}\right) \rightarrow$ $\mathscr{P}(\Sigma)$ is an injective simplicial map. Then $i\left(\mathscr{P}\left(\Sigma^{\prime}\right)\right)$ is totally geodesic $\mathscr{P}(\Sigma)$.

After applying Theorem 1.2, we see that this conjecture is equivalent to the assertion that for every multicurve $Q$ in $\Sigma, \mathscr{P}_{Q}(\Sigma)$ is totally geodesic in $\mathscr{P}(\Sigma)$. In order to state which special cases of the conjecture are known, we make several definitions. The complexity $\xi(\Sigma)$ of a compact, orientable, connected surface $\Sigma$ with genus $g$ and $b$ boundary components is $\xi(\Sigma)=3 g+b-3$, and it is a straightforward exercise to show that $\xi(\Sigma)$ is the number of curves in a pants decomposition of $\Sigma$. Given a multicurve $Q$ in $\Sigma$, we define the complementary subsurface $\Sigma_{Q}$ of $Q$ in $\Sigma$ to be the components of $\Sigma \backslash \eta(Q)$ which are not pairs of pants, where $\eta(\cdot)$ is an open regular neighborhood.

Conjecture 1 is known to be true in the case that $\xi\left(\Sigma_{Q}\right)=1$ [APS08], in the case that $\Sigma_{Q}$ has two components of complexity one, each with one 
boundary component interior to $\Sigma$ [APS09], and in the case that $\Sigma_{Q}$ is a connected surface of the same genus as $\Sigma$ [ALPS12].

We say that a multicurve $Q$ is $(n \times 1)$ if $\Sigma_{Q}$ consists of $n$ components, each having complexity 1 . The main theorem in this paper is the following:

Main Theorem. Let $Q$ be an $(n \times 1)$-multicurve. Then $\mathscr{P}_{Q}(\Sigma)$ is totally geodesic in $\mathscr{P}(\Sigma)$.

Note that if $Q$ is an $(n \times 1)$-multicurve, then $\mathscr{P}_{Q}(\Sigma)$ is a graph product of Farey graphs (see Section 3). Thus, applying Theorem $\mathrm{C}$ of [Ara10], we obtain the following corollary:

Corollary 1.1. Suppose that $G$ is a graph product of Farey graphs and $\varphi: G \rightarrow \mathscr{P}(\Sigma)$ is a simplicial embedding. Then $\varphi(G)$ is totally geodesic in $\mathscr{P}(\Sigma)$.

The rank $r$ of a metric space $X$ is defined to be the maximal $r$ such that $\mathbb{Z}^{r}$ quasi-isometrically embeds in $X$. By [BF06], [BM08], and [Ham05], the rank $r$ of $\mathscr{P}(\Sigma)$ is the maximal $n$ such that $\Sigma$ contains an $(n \times 1)$-multicurve, where $r=\left\lfloor\frac{3 g+b-2}{2}\right\rfloor$. By fixing an $(r \times 1)$-multicurve $Q$ and a bi-infinite geodesic in each of the $r$ Farey graphs composing the graph product $G=$ $\mathscr{P}_{Q}(\Sigma)$, we have another corollary:

Corollary 1.2. There exists an isometric embedding $\imath: \mathbb{Z}^{r} \rightarrow \mathscr{P}(\Sigma)$ if and only if $r \leq\left\lfloor\frac{3 g+b-2}{2}\right\rfloor$.

We remark that shortly before the completion of this note, José Estévez announced a related result [Est13]. He shows the Main Theorem holds if, in addition, one assumes that each boundary component of $\Sigma_{Q}$ is separating, and as a consequence, he gives an alternate proof of Corollary 1.2.

Acknowledgements The first author thanks Alan Reid for his ongoing support and the second author thanks Cameron Gordon for helpful conversations and insights over the course of this project.

\section{PRELIMINARIES}

Throughout this paper, $\Sigma$ will denote a compact, connected, orientable genus $g$ surface with $b$ boundary components and $3 g+b-3>0$. We occasionally use $\Sigma_{g, b}$ when we wish to emphasize $g$ and $b$, and we also call $\Sigma_{g, b}$ a $b$-times-punctured, genus $g$ surface (despite the fact that $\Sigma$ is compact). We let $\eta(\cdot)$ represent an open regular neighborhood in $\Sigma$. A loop in $\Sigma$ is a simple closed curve and the loop $c$ is essential if it is neither trivial nor peripheral. Recall that $c$ is trivial if it bounds a disk in $\Sigma$ and is peripheral 
if it is isotopic to a component of $\partial \Sigma$. We use the term curve to mean a free isotopy class $[c]$ of an essential loop $c$. For curves $\alpha$ and $\beta$, we denote by $i(\alpha, \beta)$ their (geometric) intersection number. This is by definition $\min \{|a \cap b|: a \in \alpha, b \in \beta\}$. The curves $\alpha$ and $\beta$ are disjoint if $i(\alpha, \beta)=0$; otherwise, they intersect.

A multicurve is a collection of pairwise disjoint curves in $\Sigma$. Given any collection of curves in $\Sigma$, we may always choose loop representatives that minimize pairwise geometric intersection numbers by, for example, choosing geodesic representatives in some fixed hyperbolic metric. (This is possible since $3 g+b-3>0$.) We will make such choices of representatives without further comment.

A pants decomposition $v$ of $\Sigma$ is defined to be a maximal multicurve on $\Sigma$. Its named is derived from the fact that $\Sigma \backslash \eta(v)$ is a collection of pairs of pants, i.e. copies of $\Sigma_{0,3}$. The complexity $\xi(\Sigma)$ is the cardinality $|v|$ of $v$, where $\xi(\Sigma)=3 g+b-3$. An essential subsurface $X$ of $\Sigma$ is a compact codimension- 0 submanifold such that each component of $\partial X$ is nontrivial in $\Sigma$. Note that if $X$ is an essential subsurface of $\Sigma$, then $\xi(X) \leq \xi(\Sigma)$ with equality if and only if all boundary components of $X$ are parallel to boundary components of $\Sigma$. For any multicurve $Q$, the codimension of $Q$ is defined to be $\xi(\Sigma)-|Q|$.

The pants graph $\mathscr{P}(\Sigma)$ of $\Sigma$ is the graph defined as follows: vertices are pants decompositions, and two pants decompositions $v$ and $v^{\prime}$ are connected by an edge whenever they differ by an elementary move. By this we mean that $v \cap v^{\prime}$ is a multicurve of codimension one, and letting $\gamma$ and $\gamma^{\prime}$ denote the unique curves in $v \backslash v^{\prime}$ and $v^{\prime} \backslash v$, respectively, we have that $\gamma$ and $\gamma^{\prime}$ intersect in the minimal possible number of times. Observe that $\Sigma \backslash \eta\left(v \cap v^{\prime}\right)$ is a collection of pairs of pants and a subsurface $Y$ of complexity one (which must contain $\gamma$ and $\gamma^{\prime}$ ). If $Y$ is $\Sigma_{1,1}$, the requirement of minimal intersection number implies that $i\left(\gamma, \gamma^{\prime}\right)=1$; otherwise $Y$ is $\Sigma_{0,4}$ and $i\left(\gamma, \gamma^{\prime}\right)=2$. See Figure 11. In either case, we call (the isotopy class of) $Y$ the support of the pants move from $\gamma$ to $\gamma^{\prime}$. The pants graph is connected and is equipped with a natural metric $d$ on its vertex set by assigning length one to each edge and defining the distance from $v$ to $v^{\prime}$ to be the length of the shortest path connecting $v$ to $v^{\prime}$. We note that the above definition holds if $\Sigma$ is the disjoint union of components $\left\{\Sigma_{i}\right\}$. In this case, $\mathscr{P}(\Sigma)=\mathscr{P}\left(\Sigma_{1}\right) \times \ldots \times \mathscr{P}\left(\Sigma_{n}\right)$.

Suppose that $v_{0}, v_{1}, \ldots, v_{p}$ is a path in $\mathscr{P}(\Sigma)$, and let $S_{i}$ denote the support of the $i$ th elementary move in the path, $1 \leq i \leq p-1$. Define the support $X$ 


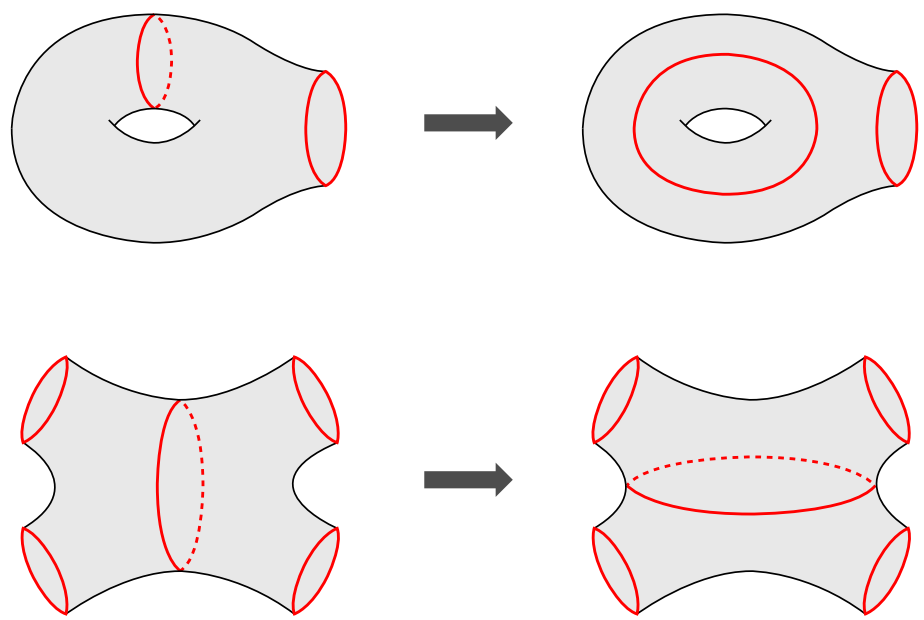

FIGURE 1. Two possible elementary moves with different supports.

of the path $v_{0}, \ldots, v_{p}$ to be

$$
X=\bigcup_{i=1}^{p-1} \overline{S_{i} \backslash \eta\left(\partial S_{i}\right)} .
$$

Thus, $v_{0} \cap X, \ldots, v_{p} \cap X$ is a path in $\mathscr{P}(X)$, and $\partial X$ is isotopic to curves in $\partial \Sigma \cup\left(v_{0} \cap v_{p}\right)$ (possibly $v_{0} \cap v_{p}=\emptyset$ and $\partial X=\partial \Sigma$ ). It is important to note that, in general, $X \neq \cup S_{i}$; this is the case in Figure 2 below.

Now, let $v_{0}, v_{1}, v_{2}$ be a path of length two in $\mathscr{P}(\Sigma)$ whose support $X$ has two connected components. Equivalently, $v_{0}, v_{1}$ and $v_{1}, v_{2}$ each differ by an elementary move with supports $S_{1}$ and $S_{2}$, respectively, such that the interiors of $S_{1}$ and $S_{2}$ are disjoint. In this case, we say that the elementary moves commute and we note that $v_{0} \cap v_{2}$ is a codimension two multicurve. Define a commutation of edges to be the path $v_{0}, v_{1}^{\prime}, v_{2}$, where $v_{1}^{\prime}=\left(v_{0} \cap v_{2}\right) \cup\left(v_{2} \backslash v_{1}\right) \cup\left(v_{0} \backslash v_{1}\right)$ is a pants decomposition since $v_{2} \backslash v_{1} \subset S_{2}$ and $v_{0} \backslash v_{1} \subset S_{1}$. In other words, the commutation of edges is the path obtained by performing the pants move supported in $S_{2}$ before the pants move supported in $S_{1}$. See Figure 2. For general edge paths in $\mathscr{P}(\Sigma)$, a commutation of edges is a sequence of commutations performed on length two subpaths. Note that if $v_{0}, \ldots, v_{p}$ is a geodesic, then any path $v_{0}, v_{1}^{\prime}, \ldots, v_{p-1}^{\prime}, v_{p}$ resulting from commutation of edges is also a geodesic with the same support as the original path. For this reason, we will often abuse notation and suppress the prime notation, renaming the new path $v_{0}, v_{1}, \ldots, v_{p-1}, v_{p}$. 


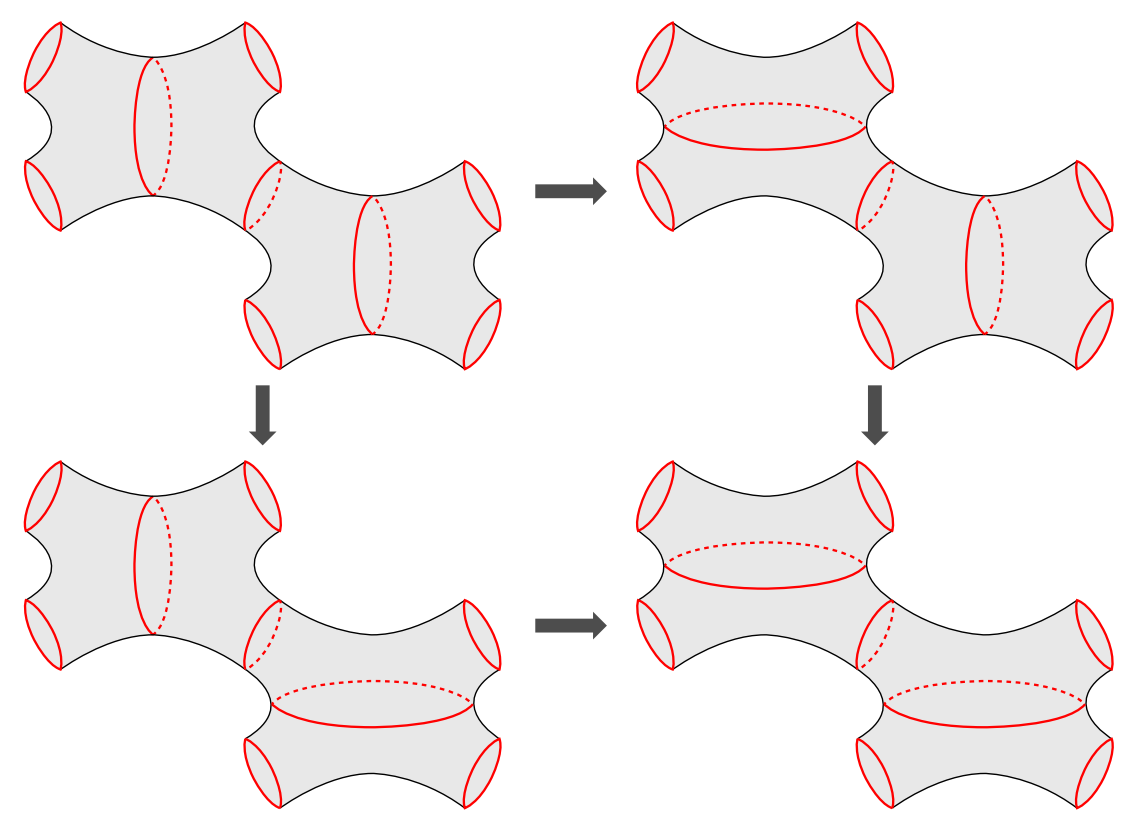

FIGURE 2. A commutation of edges in $\mathscr{P}(\Sigma)$.

Given a multicurve $Q$ in $\Sigma$, we may associate several different subsurfaces to $Q$. Recall that the complementary subsurface $Y$ of $Q$ is defined as the union of the components of $\Sigma \backslash \eta(Q)$ which are not pairs of pants (if $Q$ is a pants decomposition, then $Y=\emptyset$ ). Note that $Q$ uniquely defines $Y$, up to isotopy. Using this terminology, the complementary subsurface of an $(n \times 1)$-multicurve is by definition a disjoint union of $n$ complexity one subsurfaces. The support $X$ of a path $v_{0}, \ldots, v_{p}$ in $\mathscr{P}(\Sigma)$ is the complementary subsurface of $v_{0} \cap v_{p}$.

Let $Q$ be a multicurve in $\Sigma$ with complementary subsurface $Y$. For any pants decomposition $v_{Y}$ of $Y$, we may associate a pants decomposition $v=$ $v_{Y} \cup Q$ of $\Sigma$. This yields a natural injection $i_{Q}: \mathscr{P}(Y) \rightarrow \mathscr{P}(\Sigma)$. Recall that $\mathscr{P}_{Q}(\Sigma)$ is defined to be the full subgraph of $\mathscr{P}(\Sigma)$ spanned by those pants decompositions in $\mathscr{P}(\Sigma)$ which contain $Q$, and thus $\mathscr{P}_{Q}(\Sigma)=i_{Q}(\mathscr{P}(Y))$. For two pants decompositions $v, v^{\prime} \subset \mathscr{P}_{Q}(\Sigma)$, we denote their distance in $\mathscr{P}_{Q}(\Sigma)$ as $d_{Q}\left(v, v^{\prime}\right)$. The main result in this paper is that $\mathscr{P}_{Q}(\Sigma)$ is totally geodesic in $\mathscr{P}(\Sigma)$ when $Q$ is an $(n \times 1)$-multicurve.

We will be examining the intersections of curves on $\Sigma$ with subsurfaces of $\Sigma$, and as such we must often deal with properly embedded essential arcs. Thus, we make several more definitions pertaining to arcs. If $\alpha$ is a properly embedded essential arc in $\Sigma$ (that is, $\alpha$ is not isotopic rel $\partial$ into $\partial \Sigma$ ), we say 
$\alpha$ is a wave if $\partial \alpha$ lies in a single component of $\partial \Sigma$ or a seam if $\partial \alpha$ lies in different components of $\partial \Sigma$. When we wish to emphasize that we are interested in the isotopy class of an arc (or a collection of arcs) we use the notation $[\alpha]$.

\section{Subsurface PROJECTIONS}

A crucial tool in our proof of the main theorem is the projection of a pants decomposition $v$ of $\Sigma$ to a collection of curves in a disjoint union of complexity one subsurfaces. This projection is a special case of the MasurMinsky subsurface projections defined in [MM00]. First, suppose that $Y$ is a connected subsurface of $\Sigma$ with $\xi(Y)=1$ and that $\alpha$ is a properly embedded essential arc in $Y$. Define the projection of $\alpha$ to $Y$, denoted $\pi_{Y}(\alpha)$, to be the unique curve in $Y$ that misses $\alpha$. If $Y$ is $\Sigma_{1,1}$, then $\alpha$ is a wave and $\partial \eta(\alpha \cup \partial Y)$ has two components which are isotopic in $Y$. This curve is $\pi_{Y}(\alpha)$. If $Y$ is $\Sigma_{0,4}$ and $\alpha$ is a wave, one component of $\partial \eta(\alpha \cup \partial Y)$ is isotopic into $\partial Y$ and the other component isotopic to $\pi_{Y}(\alpha)$. Otherwise, $\alpha$ is a seam, $\partial \eta(\alpha \cup \partial Y)$ has precisely one component, which is essential in $Y$, and this component is $\pi_{Y}(\alpha)$. See Figure 3. Note that these projections depend only on the free isotopy classes of $\operatorname{arcs}$ in $Y$, and for a collection of $\operatorname{arcs} Q$ in $Y$, let $[Q]=\{[\alpha]: \alpha \in Q\}$.
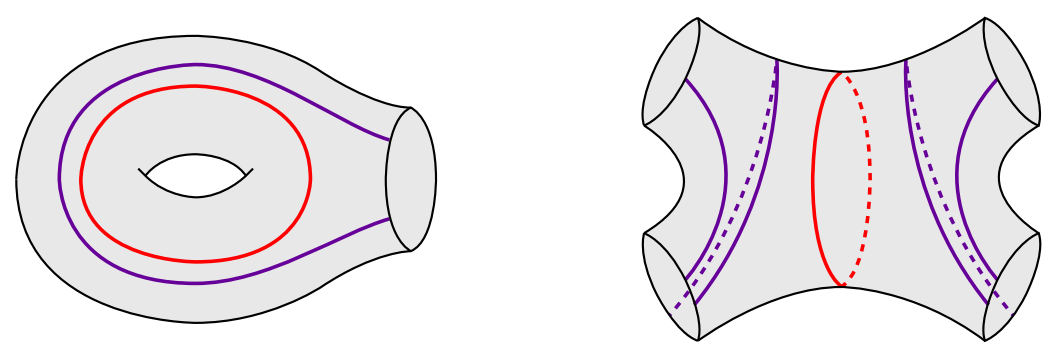

FIGURE 3. Arcs and their projection curves in $\Sigma_{1,1}$ and $\Sigma_{0,4}$.

For a curve $\beta$ in $\Sigma$, the projection $\pi_{Y}(\beta)$ is defined as follows: first represent $\partial Y$ and $\beta$ by elements with minimal geometric intersection number (if $\beta$ is isotopic to a component of $\partial Y$ choose its representative to not intersect $Y$ ). If $\beta \cap \partial Y=\emptyset$, then either $\beta \subset Y$ and we set $\pi_{Y}(\beta)=\{\beta\}$, or $\beta \subset \Sigma \backslash Y$ and we set $\pi_{Y}(\beta)=\emptyset$. Otherwise, $\beta \cap Y=\left\{b_{1}, \ldots, b_{k}\right\}$ is a nonempty collection of essential arcs in $Y$ whose isotopy classes depend only on the isotopy class of $\beta$ and set

$$
\pi_{Y}(\beta)=\bigcup_{1 \leq i \leq k} \pi_{Y}\left(b_{i}\right)
$$


For a multicurve $v$, define $\pi_{Y}(v)=\bigcup \pi_{Y}\left(v_{i}\right)$, where the $v_{i}$ are the components of $v$. If $\pi_{Y}(v) \neq \emptyset$ then we say $v$ meets $Y$, and $v$ misses or avoids $Y$ otherwise.

Suppose now that $Y$ is the disjoint union of complexity one subsurfaces $Y_{1}, \ldots, Y_{n}$ of $\Sigma$. A pants decomposition $v$ of $\Sigma$ meets each $Y_{i}$, and thus we define

$$
\pi_{Y}(v)=\left\{\left(w_{1}, \ldots, w_{n}\right) \in \mathscr{P}(Y): w_{i} \in \pi_{Y_{i}}(v)\right\} .
$$

As such, we wish to characterize the distance $d_{Y}\left(v, v^{\prime}\right)$ in $Y$ between projections of $v$ and $v^{\prime}$ in $\mathscr{P}(\Sigma)$. However, $\pi_{Y}(v)$ is a set of pants decompositions of $Y$; hence we make the following definition:

$$
d_{Y}\left(v, v^{\prime}\right)=\max _{\mu \in \pi_{Y}(v)}\left\{\min _{\mu^{\prime} \in \pi_{Y}\left(v^{\prime}\right)}\left\{d_{Y}\left(\mu, \mu^{\prime}\right)\right\}\right\}
$$

where $d_{Y}\left(\mu, \mu^{\prime}\right)$ denotes the distance between $\mu$ and $\mu^{\prime}$ in $\mathscr{P}(Y)$. If $Q$ and $Q^{\prime}$ are multicurves or collections of arcs meeting each $Y_{i}$, we may define $d_{Y}\left(Q, Q^{\prime}\right)$ similarly. We caution the reader that this distance is, in general, not symmetric. It does, however, satisfy a triangle inequality: for multicurves $Q, Q^{\prime}$, and $Q^{\prime \prime}$ meeting each $Y_{i}$, we have $d_{Y}\left(Q, Q^{\prime \prime}\right) \leq$ $d_{Y}\left(Q, Q^{\prime}\right)+d_{Y}\left(Q^{\prime}, Q^{\prime \prime}\right)$. In addition, we recall that if $\gamma$ is a curve contained in $Y_{i}$, then $\pi_{Y_{i}}(\gamma)=\{\gamma\}$; hence, if $Q$ is an $(n \times 1)$-multicurve with complementary subsurface $Y$ and $v, v^{\prime} \in \mathscr{P}_{Q}(\Sigma)$, then $\pi_{Y}(v)=\{v \cap \operatorname{int}(Y)\}$ and $d_{Y}\left(v, v^{\prime}\right)=d_{Q}\left(v, v^{\prime}\right)$.

Note that for a complexity one surface $Y$, the pants graph $\mathscr{P}(Y)$ is a Farey graph, shown in Figure 4. If $Y$ is $\Sigma_{1,1}$, an arbitrary multicurve $Q$ in $\Sigma$ intersects $Y$ in at most three isotopy classes of arcs whose projections form a geodesic triangle in $\mathscr{P}(Y)$. Thus, the diameter of $\pi_{Y}(Q)$ is at most one. If $Y$ is $\Sigma_{0,4}$, the situation is only slightly more complicated, as described below.

Lemma 3.1. Given a multicurve $Q$ in $\Sigma$ and a 4-punctured sphere $Y \subset \Sigma$, the diameter of $\pi_{Y}(Q)$ is no more than two. Further, disjoint arcs $\alpha$ and $\alpha^{\prime}$ in $Y$ satisfy $d_{Y}\left(\pi_{Y}(\alpha), \pi_{Y}\left(\alpha^{\prime}\right)\right)=2$ if and only if $\alpha$ and $\alpha^{\prime}$ are nonisotopic seams with boundary in the same two components of $\partial Y$.

Proof. Let $\alpha$ and $\alpha^{\prime}$ be disjoint nonisotopic arcs in $Y$. Each component of $\partial Y$ common to $\alpha$ and $\alpha^{\prime}$ contributes at most two points of intersection to $\pi_{Y}(\alpha) \cap \pi_{Y}\left(\alpha^{\prime}\right)$. Thus, $d\left(\pi_{Y}(\alpha), \pi_{Y}\left(\alpha^{\prime}\right)\right) \leq 1$ unless $\alpha$ and $\alpha^{\prime}$ have boundary in two common boundary components. We may verify that in this case, $\left|\pi_{Y}(\alpha) \cap \pi_{Y}\left(\alpha^{\prime}\right)\right|=4$; thus $d_{Y}\left(\pi_{Y}(\alpha), \pi_{Y}\left(\alpha^{\prime}\right)\right)=2$ (see Figure 5). If $Q$ is a multicurve in $\Sigma$, then arcs of $Q \cap Y$ are pairwise disjoint, completing the proof. 


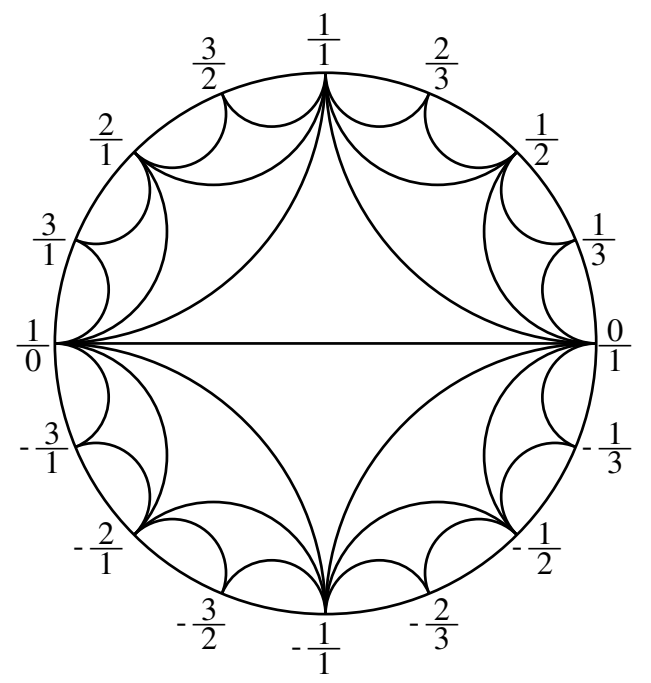

FIGURE 4. Part of a Farey graph, with edges realized as geodesics in the disk model of $\mathbb{H}^{2}$. Two vertices $a / b$ and $c / d$ are connected by an edge whenever $|a d-b c|=1$.

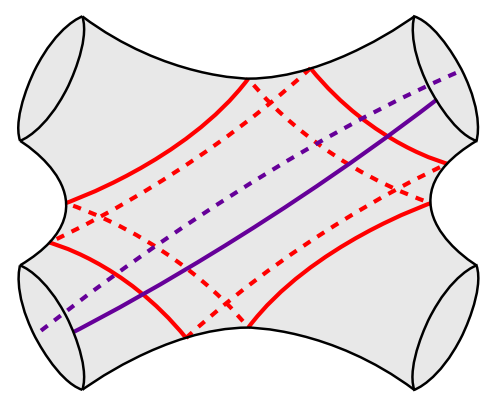

FIGURE 5. Two disjoint seams in $\Sigma_{0,4}$ that project to curves intersecting four times.

We require several technical lemmas before proceeding to the proof of the main points. From here on, we will let $\bar{\chi}(\cdot)$ denote $-\chi(\cdot)$. Suppose that $X$ and $Y$ are subsurfaces of $\Sigma$, with $X \cap Y \neq \emptyset$. We will always assume that these surfaces have been isotoped so that $\partial X$ and $\partial Y$ intersect minimally. In this context, we call $X \cap Y$ (or one of its components) a cornered subsurface. There is a cell decomposition of $X$ induced by $X \cap \partial Y$ containing $X \cap Y$ as a subcomplex, and we may count the contribution of $X \cap Y$ to $\bar{\chi}(X)$ in this cell decomposition. We define

$$
\bar{\chi}_{X}(Y)=\bar{\chi}(X \cap Y)-\frac{1}{2} \bar{\chi}\left(\operatorname{Fr}_{X} Y\right),
$$


where $\operatorname{Fr}_{X} Y$ (the frontier of $Y$ in $X$ ) denotes $X \cap \partial Y$.

Observe that the boundary components of a cornered subsurface are either curves contained in $\partial X$ or $\partial Y$ or $2 n$-gons consisting of arcs which alternate between $\operatorname{Fr}_{X}(Y)$ and $\operatorname{Fr}_{Y}(X)$. In addition, a cornered subsurface may not have any "corners;" that is, all boundary components may curves. Two particular cornered subsurfaces will be most relevant: If a component $A \subset X \cap Y$ is an annulus such that one component of $\partial A$ is contained in $X$ or $Y$ and the other component is a rectangle, we say $A$ is a rectangular annulus. Similarly, If $P \subset X \cap Y$ is a pair of pants such that two components of $\partial P$ are contained in $X$ or $Y$ and the other component is a rectangle, we say $P$ is a rectangular pair of pants. Note that $\bar{\chi}_{X}(A)=1$ and $\bar{\chi}_{X}(P)=2$. See Figure 6
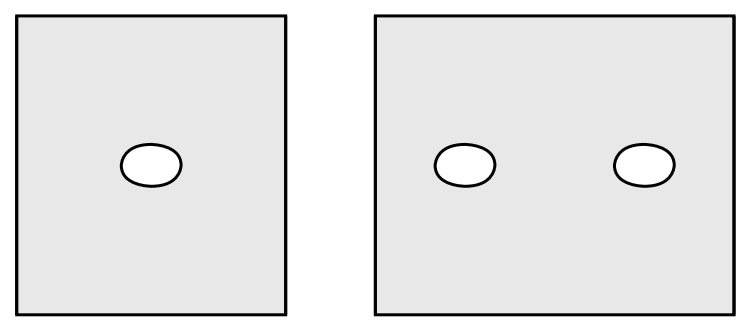

FIGURE 6. A rectangular annulus and a rectangular pair of pants.

Lemma 3.2. For subsurfaces $X$ and $Y$ of $\Sigma$, we have $\bar{\chi}_{X}(Y) \leq \bar{\chi}(X)$.

Proof. Consider a cell decomposition of $X$ induced by $X \cap \partial Y$ containing $X \cap Y$ as a subcomplex, and let $\left\{Z_{i}\right\}$ denote the closures of the components of $X \backslash \partial Y$. Thus, each $Z_{i}$ inherits a cell decomposition, and either $Z_{i} \subset X \cap Y$ or $Z_{i} \subset X \backslash Y$. Let $f$ denote the number of faces in this decomposition, $v_{F}$ and $e_{F}$ the numbers of vertices and edges (respectively) contained in $\operatorname{Fr}_{X}(Y)$, and $v_{Z}$ and $e_{Z}$ the numbers of vertices and edges (respectively) not contained in $\operatorname{Fr}_{X}(Y)$. Note that if we compute $\sum \bar{\chi}\left(Z_{i}\right)$ and $\sum \bar{\chi}\left(\operatorname{Fr}_{X} Z_{i}\right)$, vertices and edges contained in $\operatorname{Fr}_{X}(Y)$ are counted twice, while faces and all other vertices and edges are counted once. Thus

$$
\begin{aligned}
\sum \bar{\chi}_{X}\left(Z_{i}\right) & =\sum \bar{\chi}\left(Z_{i}\right)-\frac{1}{2} \sum \bar{\chi}\left(\operatorname{Fr}_{X} Z_{i}\right) \\
& =\left(-2 v_{F}-v_{Z}+2 e_{F}+e_{Z}-f\right)-\frac{1}{2}\left(-2 v_{F}+2 e_{F}\right) \\
& =-v_{F}-v_{Z}+e_{F}+e_{Z}-f \\
& =\bar{\chi}(X) .
\end{aligned}
$$


Requiring that $X$ and $Y$ are essential subsurfaces and $|\partial X \cap \partial Y|$ is minimal up to isotopy ensures that $\bar{\chi}_{X}\left(Z_{i}\right) \geq 0$ for all $i$. It follows that

$$
\bar{\chi}_{X}(Y)=\sum_{Z_{i} \subset Y} \bar{\chi}_{X}\left(Z_{i}\right) \leq \sum \bar{\chi}_{X}\left(Z_{i}\right) \leq \bar{\chi}(X)
$$

as desired.

From the proof of the lemma, we may also conclude that $\bar{\chi}_{X}(Y)=\bar{\chi}(X)$ if and only if $\bar{\chi}_{X}(X \backslash \cup \eta(Y))=0$. See Figure 7

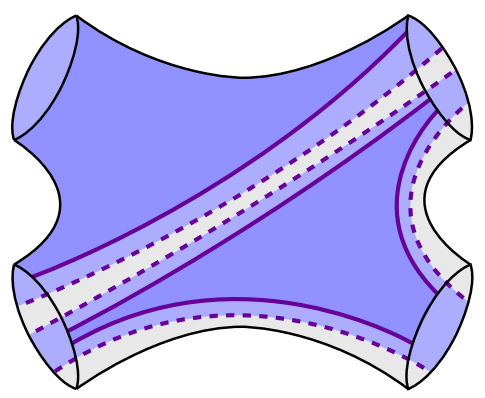

FIGURE 7. A 4-punctured sphere $X$ containing a rectangular annulus $D_{1}$ and two hexagons $D_{2}$ and $D_{3}$. Note that $\bar{\chi}(X)=\bar{\chi}_{X}\left(D_{1}\right)+\bar{\chi}_{X}\left(D_{2}\right)+\bar{\chi}_{X}\left(D_{3}\right)$ in this example.

In the next two lemmas, we examine the contribution of a complexity one subsurface $Y$ to the Euler characteristic of another subsurface $X$ under some assumptions on the distance from $\pi_{Y}(c)$ to $\pi_{Y}(\partial X)$ for a curve $c$ in $X$. These lemmas will later be used to compare the support $X$ of a path in $\mathscr{P}(\Sigma)$ to the complementary subsurface $Y$ of an $(n \times 1)$-multicurve.

Lemma 3.3. Suppose that $X$ and $Y$ are subsurfaces of $\Sigma$, with $\xi(Y)=1$ and $\partial X \cap Y \neq \emptyset$. Let $c$ be a curve contained in $X$ which meets $Y$. If $d_{Y}(c, \partial X)=$ 1 , then there is a cornered component $D$ of $X \cap Y$ such that $\bar{\chi}_{X}(D) \geq 1$.

Proof. The key observation here is that if every component $D$ of $X \cap Y$ is a rectangle or a hexagon, then $c$ satisfies $\pi_{Y}(c) \subset \pi_{Y}(\partial X)$, as any arc of $c \cap D$ is isotopic in $Y$ to an arc in $\partial X \cap D$. Hence, if all components of $X \cap Y$ are $2 n$-gons, there is a $2 n$-gon $D$ with $n \geq 4$; hence $\bar{\chi}_{X}(D) \geq 1$. See Figure 8 ,

If there is a component $D$ of $X \cap Y$ which is not a topological disk, then $D$ is a punctured sphere or torus, and if $D$ has three or more punctures or is not planar, then $\bar{\chi}_{X}(D) \geq \bar{\chi}(D) \geq 1$. Thus, suppose that $D$ is an annulus. If $\partial D$ contains two or more $\operatorname{arcs}$ in $\operatorname{Fr}_{X}(Y)$, then $\bar{\chi}_{X}(D) \geq 2 \cdot \frac{1}{2}$. Otherwise, one component of $\partial D$ is the union of an $\operatorname{arc}$ in $\operatorname{Fr}_{X}(Y)$ and a wave $\alpha \subset Y$ 


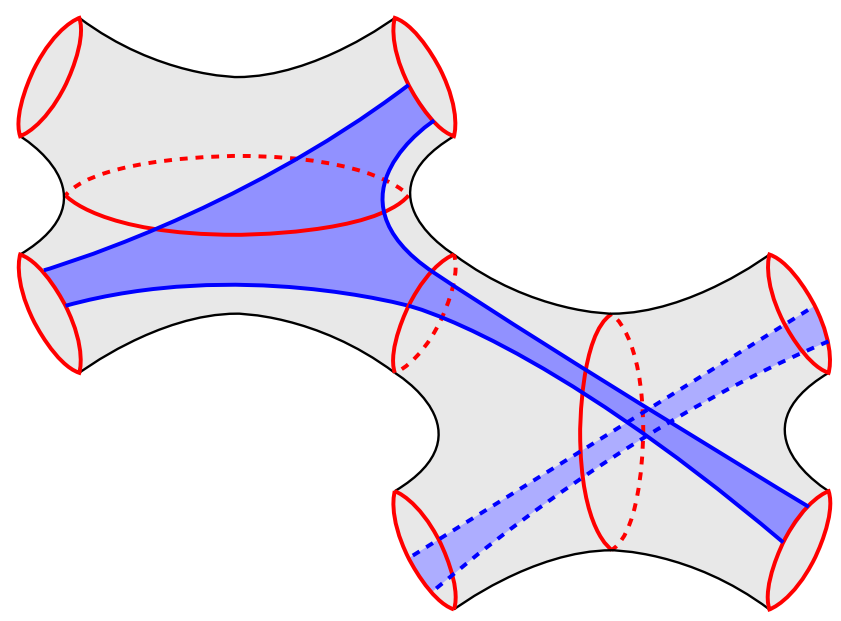

FIgURE 8 . Components $D_{1}$ and $D_{2}$ of $X \cap Y$ in $X$, where $D_{1}$ is a hexagon and $D_{2}$ is a rectangle. For an interior curve $c$, any component of $c \cap D_{i}$ is parallel in $Y$ to a component of $\partial X \cap Y$.

and the other component is a curve $\gamma$ in $X$. If $\gamma$ is essential in $Y$, then $\pi_{Y}(c)=\pi_{Y}(\partial X)=\{\gamma\}$, a contradiction. Thus, $\gamma$ is isotopic into $\partial Y$, and it follows that $|\partial Y|>1$, so $Y$ is a 4-punctured sphere. However, if $c$ intersects $D$, then each component $\beta$ of the intersection $c \cap D$ is an essential arc with the property that $\pi_{Y}(\alpha)=\pi_{Y}(\beta)$ and $d_{Y}(c, \partial X)=0$, a contradiction. See Figure 9 .

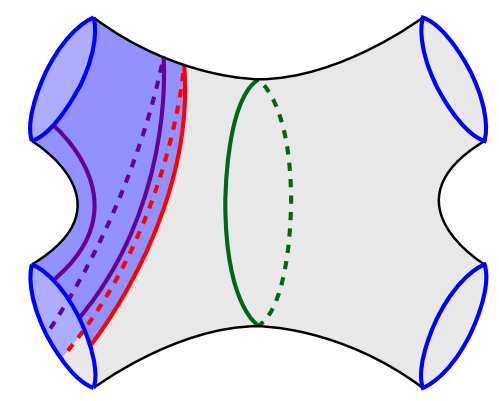

FIGURE 9. A depiction in $Y$ of the case in which $D$ is an annulus and $\partial D$ contains one $\operatorname{arc}$ of $\operatorname{Fr}_{X}(Y)$. Note that all arcs pictured project to the same curve in $Y$.

Lemma 3.4. Suppose that $X$ and $Y$ are subsurfaces of $\Sigma$ with $Y$ a 4-punctured sphere and $\partial X \cap Y \neq \emptyset$. Let $c$ be a curve contained in $X$. If $d_{Y}(c, \partial X)=2$, 
then there is a cornered component $D$ of $X \cap Y$ which is a rectangular pair of pants.

Proof. By Lemma 3.1. some arc $\alpha$ of $c \cap Y$ is a seam, and every arc of $\partial X \cap Y$ is a seam not isotopic to $\alpha$ but with endpoints on the same two components $\gamma_{1}$ and $\gamma_{2}$ of $\partial Y$ as $\alpha$. Any two distinct classes of arcs which are disjoint from $\alpha$ with endpoints on $\gamma_{1}$ and $\gamma_{2}$ must intersect, and thus all arcs of $\partial X \cap Y$ are isotopic in $Y$. Cutting $Y$ along the arcs of $\partial X \cap Y$ yields some number of rectangles and the desired component $D$.

We present three final technical lemmas before proceeding to the proof of the main theorems.

Lemma 3.5. Let $P$ be a pair of pants and $Z$ a subsurface of $\Sigma$. Let $D$ be a cornered component of their intersection. If $D$ is an octagon then $D$ intersects all three components of $\partial P$, and if $D$ is a rectangular annulus then $D$ intersects two components of $\partial P$.

Proof. First let $D$ be an octagon. Suppose by way of contradiction that $D$ avoids a boundary component $p$ of $P$. Then $P \backslash \eta(D)$ has a component $C$ which contains $p$. It follows that $\bar{\chi}(P) \geq \bar{\chi}_{P}(D)+\bar{\chi}_{P}(C)>1+0$, a contradiction to Lemma 3.2 .

Now let $D$ be a rectangular annulus. First, note that the curve boundary component of $D$ is isotopic into $\partial P$, so $D$ can intersect at most two components of $\partial P$. Suppose by way of contradiction that $D$ avoids two boundary components of $P$. Then one of them, call it $p$, is not isotopic to the curve boundary component of $D$, so $P \backslash \eta(D)$ has a component $C$ which contains p. It follows that $\bar{\chi}(P) \geq \bar{\chi}_{P}(D)+\bar{\chi}_{P}(C)>1+0$, a contradiction.

Lemma 3.6. Let $X \subset \Sigma$ be a subsurface containing two curves $\gamma_{1}$ and $\gamma_{2}$. Suppose $Y$ is a 4-punctured sphere such that $X \cap Y$ contains a rectangular annulus $A$, where both $\gamma_{1}$ and $\gamma_{2}$ meet $A$. Then

$$
\max \left\{d_{Y}\left(w_{1}, w_{2}\right): w_{i} \in \pi_{Y}\left(\gamma_{i} \cap A\right)\right\} \leq 2 .
$$

Proof. Up to isotopy, there are up to four possible seams and two possible waves contained in $\gamma_{i} \cap A$. See Figure 10, Let $\alpha, \beta \subset \partial A$ denote the arcs contained in $\partial X$. By Lemma 3.1 $d_{Y}\left(\pi_{Y}(\alpha), \pi_{Y}(\beta)\right)=2$, and for any arc $\delta_{i} \subset \gamma_{i} \cap A$ which is not isotopic to $\alpha$ or $\beta$, we have $d_{Y}\left(\pi_{Y}\left(\delta_{i}\right), \pi_{Y}(\alpha)\right) \leq 1$. Thus for any two such arcs $\delta_{1} \subset \gamma_{1} \cap A$ and $\delta_{2} \subset \gamma_{2} \cap A$,

$$
d_{Y}\left(\pi_{Y}\left(\delta_{1}\right), \pi_{Y}\left(\delta_{2}\right)\right) \leq d_{Y}\left(\pi_{Y}\left(\delta_{1}\right), \pi_{Y}(\alpha)\right)+d_{Y}\left(\pi_{Y}(\alpha), \pi_{Y}\left(\delta_{2}\right)\right) \leq 2 .
$$




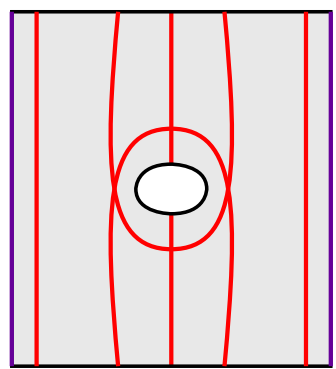

FIgURE 10. The six possibilities for arcs of $\gamma_{i} \cap A$ in $A$.

Lemma 3.7. Let $X \subset \Sigma$ be an essential subsurface such that $v$ and $v^{\prime}$ are pants decompositions of $X$ related by an elementary move. Suppose $Y$ is a 4-punctured sphere such that $X \cap Y$ contains a rectangular pair of pants $P$, where $p_{1}$ and $p_{2}$ denote the curve boundary components of $P$. If $\delta$ and $\delta^{\prime}$ are arc components of $v \cap P$ and $v^{\prime} \cap P$ (respectively) that avoid both $p_{1}$ and $p_{2}$, then $d_{Y}\left(\pi_{Y}(\delta), \pi_{Y}\left(\boldsymbol{\delta}^{\prime}\right)\right) \leq 2$.

Proof. Let $w=\pi_{Y}(\delta)$ and $w^{\prime}=\pi_{Y}\left(\delta^{\prime}\right)$, and note that $\partial X \cap Y$ contains a single class of arcs in $Y$, a seam $[\alpha]$. If both $\delta$ and $\delta^{\prime}$ are waves or are isotopic to $\alpha$, then by Lemma 3.1 ,

$$
d_{Y}\left(w, w^{\prime}\right) \leq d_{Y}\left(w, \pi_{Y}(\alpha)\right)+d_{Y}\left(\pi_{Y}(\alpha), w^{\prime}\right) \leq 1+1=2 .
$$

Thus, suppose without loss of generality that $\delta^{\prime}$ is a seam distinct from $\alpha$ and consider the possibilities for $\delta$, observing that $\alpha \cap \delta=\emptyset$. If $\delta \cap \delta^{\prime}=\emptyset$, the statement holds by Lemma 3.1. Otherwise, $\delta \cap \delta^{\prime}$ can be at most two points with signed intersection at most \pm 1 . We leave it to the reader to verify that, up to a homeomorphism of $P$, there is precisely one such seam $\delta_{1}$ and two such waves $\delta_{2}$ and $\delta_{3}$ which are candidates for $\delta$. See Figure 11
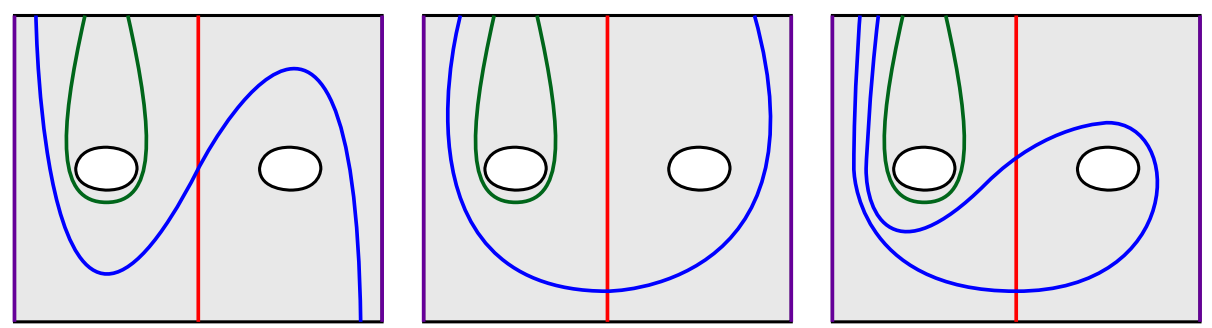

FIGURE 11. Three possibilities for a seam (left) or wave (middle and right) $\delta$. Note that in each case there is a wave $\varepsilon$ disjoint from $\delta$ and $\delta^{\prime}$. 
However, we note that for each $\delta_{i}$ we may find a wave $\varepsilon$ disjoint from $\delta_{i} \cup \delta^{\prime}$, implying by Lemma 3.1 that $d_{Y}\left(w, w^{\prime}\right) \leq 2$. This completes the proof.

\section{Proof of THE MAIN THEOREM}

In order to prove the main theorem, we first turn to the proof of Theorem 4.1. We will not require the full generality of Theorem 4.1, however, it may be of independent interest. It provides a Lipschitz property for the projection of a path in $\mathscr{P}(\Sigma)$ to $\mathscr{P}(Y)$, where $Y$ is the complementary subsurface of an $(n \times 1)$-multicurve. The interested reader may compare Theorem 4.1 to Theorem 2 of [APS08] or Theorem 2 of [APS09].

Theorem 4.1. Suppose that $Q$ is an $(n \times 1)$-multicurve in $\Sigma$ such that $Y=$ $\Sigma_{Q}$, and let $v_{0}, \ldots, v_{p}$ denote a path in $\mathscr{P}(\Sigma)$ such that $p \geq \bar{\chi}(Y)$. After a possible commutation of edges, there exists $q$ such that $0<q \leq p$ and

$$
d_{Y}\left(v_{0}, v_{q}\right) \leq q .
$$

The proof of Theorem 4.1 is divided into Lemmas 4.1 and 4.2, For both lemmas, we suppose $Y=\cup_{i=1}^{n} Y_{i}$, where $\xi\left(Y_{i}\right)=1$. In addition, let $S_{j}$ denote the support of the $j$ th elementary move and $X_{j}$ denote the support of the path $v_{0}, \ldots, v_{j}$. Note that $X_{0}=\emptyset \subset X_{1} \subset \cdots \subset X_{p} \subset \Sigma$.

Lemma 4.1. If $X_{p}$ does not contain a component $Y_{i}$ of $Y$, then

$$
d_{Y}\left(v_{0}, v_{p}\right) \leq p .
$$

Proof. If $X_{p} \cap Y_{i}=\emptyset$, then $v_{0} \cap Y_{i}=v_{p} \cap Y_{i}$ and $d_{Y_{i}}\left(v_{0}, v_{p}\right)=0$. Otherwise $X_{p} \cap Y_{i} \neq \emptyset$ and $Y_{i}$ contains an arc or curve $\alpha \subset \partial X_{p} \subset v_{0} \cap v_{p}$. Observing that $d_{Y_{i}}\left(v_{0}, v_{p}\right) \leq d_{Y_{i}}\left(v_{0}, \pi_{Y_{i}}(\alpha)\right)$, we may invoke Lemma 3.1, which implies that $d_{Y_{i}}\left(v_{0}, v_{p}\right) \leq 1$ if $Y_{i}=\Sigma_{1,1}$ and $d_{Y_{i}}\left(v_{0}, v_{p}\right) \leq 2$ if $Y_{i}=\Sigma_{0,4}$. Thus,

$$
\begin{aligned}
d_{Y}\left(v_{0}, v_{p}\right) & =\sum_{Y_{i}=\Sigma_{1,1}} d_{Y_{i}}\left(v_{0}, v_{p}\right)+\sum_{Y_{i}=\Sigma_{0,4}} d_{Y_{i}}\left(v_{0}, v_{p}\right) \\
& \leq \sum_{Y_{i}=\Sigma_{1,1}} 1+\sum_{Y_{i}=\Sigma_{0,4}} 2=\bar{\chi}(Y) \leq p,
\end{aligned}
$$

as desired.

Lemma 4.2. If there exists $p^{\prime} \leq p$ such that $X_{p^{\prime}}$ contains a component $Y_{i}$ of $Y$, then, after a possible commutation of edges, there exists $q$ such that $0<q \leq p^{\prime}$ and

$$
d_{Y}\left(v_{0}, v_{q}\right) \leq q .
$$

Proof. Choose an index $m<p^{\prime}$, such that $X_{m}$ does not contain a component $Y_{i}$ of $Y$ but $X_{m+1}$ contains some $Y_{i}$. By commuting edges, we may further assume that $X_{m+1}$ is connected. Note that if $m=0$, then $X_{m+1}=Y_{i}$ for some 
$i$ and letting $q=1$ completes the proof of the theorem. Thus, we may suppose that $m \geq 1$.

Let $\gamma_{j}$ and $\gamma_{j}^{\prime}$ denote the unique curves in $v_{j-1} \backslash v_{j}$ and $v_{j} \backslash v_{j-1}$, respectively, so that the $j$ th elementary move replaces $\gamma_{j} \in v_{j-1}$ with $\gamma_{j}^{\prime} \in v_{j}$. For any $j, 1 \leq j \leq m$, suppose for the moment that $X=X_{j}$ is connected, noting that $\xi(X)=\left|v_{0} \backslash v_{j}\right| \leq j$. In addition, if $g$ is the genus of $X$ and $b=|\partial X|$, then $\xi(X)=3 g+b-3$, whereas $\bar{\chi}(X)=2 g+b-2$. Thus, $\bar{\chi}(X) \leq \xi(X)+1$, with equality if and only if $g=0$.

By assumption, no $Y_{i}$ is contained in $X$, so for each $Y_{i}$ that meets $X$, we have $d_{Y_{i}}\left(v_{0}, v_{j}\right) \leq 2$ as in the proof of Lemma 4.1. It follows that we may partition $\left\{Y_{i}\right\}$ into

$$
\begin{aligned}
& T_{0}=\left\{Y_{i}: d_{Y_{i}}\left(v_{0}, v_{j}\right)=0\right\}, \\
& T_{1}=\left\{Y_{i}: d_{Y_{i}}\left(v_{0}, v_{j}\right)=1\right\}, \\
& T_{2}=\left\{Y_{i}: d_{Y_{i}}\left(v_{0}, v_{j}\right)=2\right\},
\end{aligned}
$$

and thus $d_{Y}\left(v_{0}, v_{j}\right)=\left|T_{1}\right|+2\left|T_{2}\right|$. Let $c_{i}$ denote any component of $v_{0}$ satisfying $d_{Y_{i}}\left(c, v_{j}\right)=d_{Y_{i}}\left(v_{0}, v_{j}\right)$. If $Y_{i} \in T_{1}, T_{2}$, then $c_{i} \subset \operatorname{int}(X)$. Further, $\partial X \subset v_{j}$ and thus $d_{Y_{i}}\left(c_{i}, v_{j}\right) \leq d_{Y_{i}}\left(c_{i}, \partial X\right)$. It follows from Lemmas 3.3 and 3.4 that $\bar{\chi}_{X}\left(Y_{i}\right) \geq 1$ if $Y_{i} \in T_{1}$ and $\bar{\chi}_{X}\left(Y_{i}\right) \geq 2$ if $Y_{i} \in T_{2}$. Therefore $\left|T_{1}\right|+2\left|T_{2}\right| \leq \sum \bar{\chi}_{X}\left(Y_{i}\right) \leq \bar{\chi}(X)$, and stringing inequalities together yields

$$
d_{Y}\left(v_{0}, v_{j}\right)=\left|T_{1}\right|+2\left|T_{2}\right| \leq \bar{\chi}(X) \leq \xi(X)+1 \leq j+1 .
$$

Of course, it may be the case that for some $j^{\prime}$ with $1 \leq j^{\prime} \leq m$, the subsurface $X_{j^{\prime}}$ of $\Sigma$ is not connected. However, for each connected component $X$ of $X_{j^{\prime}}$, we have that $X$ is the union of supports of elementary moves, so we may perform a commutation of edges so that $X=X_{j}$ for some $j \leq j^{\prime}$. (Here, $X_{j}$ is the union of the first $j$ elementary moves occurring in our new path of pants decompositions.) It follows that for any connected component $X\left(=X_{j}\right)$ of $X_{j^{\prime}}$, if any of the inequalities in (1) is not sharp, the theorem is proved with $q=j$. Thus, we may suppose for the remainder of the proof that for such $X$,

(1) $\left|T_{1}\right|+2\left|T_{2}\right|=\bar{\chi}(X)$, so that $\bar{\chi}_{X}\left(Y_{i}\right)=k$ for all $Y_{i} \in T_{k}(k=0,1,2)$,

(2) $\bar{\chi}(X)=\xi(X)+1$, so that $X$ is planar, and

(3) $\xi(X)=j$, so that $\gamma_{k}^{\prime} \neq \gamma_{l}$ for any $k, l \leq j$.

We break the remainder of the proof into a number of possibly overlapping cases, which in total will exhaust all possibilities, proving the statement in question.

Case 1. There exists $j \leq m$ such that $X_{j} \cap \partial Y$ contains a wave. 
Proof. Let $X=X_{j}$ and suppose that for some $i$, there exists $\gamma \subset \partial Y_{i}$ such that $\gamma \cap X$ contains a wave $\delta$. By the above arguments, we may assume $X$ is connected after commuting edges in $\mathscr{P}(\Sigma)$. Since $X$ is planar, $\delta$ separates $X$ into two subsurfaces $X^{\prime}$ and $X^{\prime \prime}$ such that $\bar{\chi}_{X}\left(X^{\prime}\right) \equiv \bar{\chi}_{X}\left(X^{\prime \prime}\right) \equiv \frac{1}{2}(\bmod 1)$. However, for every $i$, we have by our above assumptions on $X$ that each $Y_{i}$ contributes a component of integral Euler characteristic to $X$. It follows that either $\bar{\chi}_{X}\left(X^{\prime} \backslash \bigcup\left\{Y_{i}\right\}\right)$ or $\bar{\chi}_{X}\left(X^{\prime \prime} \backslash \bigcup\left\{Y_{i}\right\}\right)$ is positive; hence $\left|T_{1}\right|+2\left|T_{2}\right|<$ $\bar{\chi}(X)$ and the theorem is proved.

If a cornered component $D$ of $X_{j} \cap Y_{i}$ has a bigon boundary component, then $X_{j} \cap \partial Y_{i}$ contains a wave and the theorem holds by Case 1 . Thus, we may assume from this point forward that no $D$ has a bigon boundary component. For any $j$, with $1 \leq j \leq m$, suppose that $X_{j} \cap Y_{i}$ contains a pair of pants component $P$ such that $\partial P \subset \partial X_{j} \cup \partial Y_{i}$. We call such $P$ a full pair of pants.

Case 2. There is a $j$ with $1 \leq j \leq m$ such that $X_{j} \cap Y$ contains a full pair of pants.

Proof. Suppose $X_{j}$ is connected and $X_{j} \cap Y_{i}$ contains a full pair of pants $P$. Then a component $\gamma$ of $\partial X_{j}$ is essential in $Y_{i}$, implying that $\pi_{Y_{i}}\left(v_{0}\right)=$ $\pi_{Y_{i}}\left(v_{j}\right)=\{\gamma\}$. Hence $d_{Y_{i}}\left(v_{0}, v_{j}\right)=0$ but $\bar{\chi}_{X_{j}}\left(Y_{i}\right) \geq 1$, and again $\left|T_{1}\right|+$ $2\left|T_{2}\right|<\bar{\chi}\left(X_{j}\right)$.

Case 3. The curve $\gamma_{m+1}=v_{m} \backslash v_{m+1}$ is separating in $X_{m+1}$.

Proof. Suppose without loss of generality that $Y_{1} \subset X_{m+1}$. If $X_{m} \cap \partial Y_{1}=\emptyset$, then $X_{m} \cap Y_{1}=\emptyset$, as the only nontrivial subsurfaces of $Y_{1}$ have at least one boundary component isotopic into $\partial Y_{1}$. Thus, $X_{m+1}$ is the disjoint uniont of $X_{m}$ and $S_{m+1}$ and $Y_{1} \subset S_{m+1}$. In this case we may commute edges in $\mathscr{P}(\Sigma)$ so that $Y_{1} \subset S_{1}$ and $m=0$, which is addressed above. Thus, suppose $X_{m} \cap \partial Y_{1} \neq \emptyset$. If $\partial Y_{1} \subset X_{m}$, then either $Y_{1}$ is contained in a component of $X_{m}$ (which we have assumed does not occur) or $X_{m} \cap Y_{1}$ contains a full pair of pants $P$ (which refers us to Case 2). Hence, assume that $\partial X_{m} \cap \partial Y_{1} \neq \emptyset$. Since $\partial Y_{1} \subset X_{m+1}$, we have that if $\gamma_{m+1}$ separates $X_{m+1}$, then there is a component $X^{\prime}$ of $X_{m}$ such that $\gamma_{m+1}$ is isotopic in $\Sigma$ to a single component $\gamma$ of $\partial X^{\prime}$, and $\partial X^{\prime} \cap \partial Y_{1} \subset \gamma \cap \partial Y_{1}$, implying that $X^{\prime} \cap \partial Y_{1}$ contains a wave as in Case 1 .

By ruling out Case 3, we may assume from this point forward that $\gamma_{m+1}$ is nonseparating in $X_{m+1}$, and, as a consequence, $X_{m}$ is connected and $\gamma_{m+1}$ is isotopic in $\Sigma$ to two distinct components of $\partial X_{m}$. In addition, $\bar{\chi}\left(X_{m}\right)=\bar{\chi}\left(X_{m+1}\right), \gamma_{k}^{\prime} \neq \gamma_{l}$ for any $k, l \leq m$, and by (1) above, we have $d_{Y}\left(v_{0}, v_{m}\right) \leq m+1$. Recall our assumption that $Y_{1}$ is contained in $X_{m+1}$. 
Case 4. $Y_{1} \subset X_{m+1}$ is a punctured torus.

Proof. Since $\bar{\chi}_{X_{m}}\left(Y_{1}\right)=\bar{\chi}_{X_{m+1}}\left(Y_{1}\right)=1$, our previous assumptions imply $d_{Y_{1}}\left(v_{0}, v_{m}\right)=1$ and there is a component $R$ of $X_{m} \cap Y_{1}$ such that $\bar{\chi}_{X_{m}}(R)=1$. If $R$ is a full pair of pants, we refer to Case 2. Since $\left|\partial Y_{1}\right|=1, R$ is not a rectangular annulus; hence suppose that $R$ is an octagon. If $X_{m} \cap \partial Y_{1}$ contains a wave, refer to Case 1 . Otherwise $R \cap \partial Y_{1}$ consists of four seams in $X_{m}$ connecting the two components $\gamma^{\prime}$ and $\gamma^{\prime \prime}$ of $\partial X_{m}$ that are isotopic to $\gamma_{m+1}$. Any two of these seams separate the planar surface $X_{m}$; hence four such seams cannot cobound an octagon $R$.

Case 5. $Y_{1} \subset X_{m+1}$ is a 4-punctured sphere.

Proof. The bulk of the proof of the theorem will be devoted to this case, which can easily be seen to exhaust all possibilities. Since $\bar{\chi}_{X_{m}}\left(Y_{1}\right)=$ $\bar{\chi}_{X_{m+1}}\left(Y_{1}\right)=2$, our previous assumptions imply $d_{Y_{1}}\left(v_{0}, v_{m}\right)=2$ and there is a component $R$ of $X_{m} \cap Y_{1}$ such that $\bar{\chi}_{X_{m}}(R)=2$. Lemma 3.4 asserts that $R$ is a rectangular pair of pants such that $\partial X_{m} \cap Y_{1}$ contains a single class of arc, a seam $[\alpha]$. Let $q_{1}$ and $q_{2}$ denote the curve components of $\partial R$ contained entirely in $X_{m}$, noting that $q_{1}$ and $q_{2}$ are isotopic to curves in $\partial Y_{1}$.

By arguments in the proof of Lemma4.1, we have $d_{Y_{i}}\left(v_{0}, v_{m+1}\right) \leq \bar{\chi}_{X_{m+1}}\left(Y_{i}\right)$ if $Y_{i}$ is not contained in $X_{m+1}$. If for every $i, d_{Y_{i}}\left(v_{0}, v_{m+1}\right) \leq \bar{\chi}_{X_{m+1}}\left(Y_{i}\right)$ then as in (1) above,

$$
d_{Y}\left(v_{0}, v_{m+1}\right) \leq \bar{\chi}\left(X_{m+1}\right)=\bar{\chi}\left(X_{m}\right) \leq \xi\left(X_{m}\right)+1 \leq m+1,
$$

completing the proof. Otherwise, we may suppose without loss of generality that for, say, $Y_{1}$ we have $d_{Y_{1}}\left(v_{0}, v_{m+1}\right)>\bar{\chi}_{X_{m+1}}\left(Y_{1}\right)$. It follows that $Y_{1}$ must be contained in $X_{m+1}$, and we choose $Y_{1}$ so that $d_{Y_{1}}\left(v_{0}, v_{m+1}\right)$ is maximal over $\left\{Y_{i}\right\}$. Note that $\gamma_{m+1} \in v_{m}$ intersects $R$ in two arc boundary components isotopic to $\alpha$ in $Y_{1}$. By assumption, every curve of $v_{m} \cap \operatorname{int}(R)$ is one of the curves $\gamma_{k}^{\prime}$, where $k \leq m$, and as $\gamma_{k}^{\prime} \neq \gamma_{m+1}$, we have $\gamma_{k}^{\prime} \in v_{m+1}$. If some $\gamma_{k}^{\prime}$ meets $R$ in an arc or curve $\delta$, then $\delta$ is also a component of $v_{m+1} \cap Y_{1}$. We will show that either one of the previous cases holds, or there is such a $\gamma_{k}^{\prime}$ and $\delta \subset \gamma_{k}^{\prime} \cap R$ satisfying $d_{Y_{1}}\left(v_{0}, \delta\right) \leq 2$. In this case, $d_{Y_{1}}\left(v_{0}, v_{m+1}\right) \leq 2$ and (2) holds, completing the proof.

Suppose that for some $j<m, q_{1} \cap X_{j}$ contains an arc. Since $q_{1} \subset X_{m}$, we may choose $j$ such that $q_{1} \cap X_{j}$ contains an arc but $q_{1}$ is contained entirely within $X_{j+1}$. As $X_{j+1}$ is planar, $\gamma_{j}$ is separating in $X_{j+1}$, and there is a component $X^{\prime}$ of $X_{j}$ such that $\gamma_{j}$ is isotopic to a component of $\partial X^{\prime}$ containing $q_{1} \cap \partial X^{\prime}$. Hence, $q_{1} \cap X^{\prime}$ contains a wave, and we refer to Case 1 A parallel 
argument shows the same to be true if $q_{2} \cap X_{j}$ contains an arc.

We now undertake a careful analysis of the stages at which $d_{Y_{1}}\left(v_{0}, v_{j}\right)$ grows with $j$. To this end, let $r_{1}$ be the smallest index for which $d_{Y_{1}}\left(v_{0}, v_{r_{1}}\right)>$ 0 . This implies $\bar{\chi}_{X_{r_{1}-1}}\left(Y_{1}\right)=0$ and $\pi_{Y_{1}}\left(v_{0}\right) \subset \pi_{Y_{1}}\left(v_{r_{1}-1}\right)$. After commutations, we may assume that $X_{r_{1}-1}$ is connected. Suppose first that $d_{Y_{1}}\left(v_{0}, v_{r_{1}}\right)=$ 2. Then $\bar{\chi}_{X_{r_{1}}}\left(Y_{1}\right)=2$; hence $\bar{\chi}\left(X_{r_{1}} \backslash X_{r_{1}-1}\right) \geq 2$, which implies that $X_{r_{1}}$ is the disjoint union of $X_{r_{1}-1}$ and $S_{r_{1}}$. Thus, $S_{r_{1}} \cap Y_{1}$ is a rectangular pair of pants, $q_{1}$ and $q_{2}$ are isotopic into $\partial S_{r_{1}}$, and $\gamma_{r_{1}}^{\prime}$ meets $R$ in an arc or curve $\delta$. Applying Lemma 3.7 with $X=X_{r_{1}}$, for any component $\delta^{\prime}$ of $v_{r_{1}-1} \cap Y_{1}$, we have $d_{Y_{1}}\left(\delta^{\prime}, \delta\right) \leq 2$. Thus, $d_{Y_{1}}\left(v_{0}, \delta\right) \leq d_{Y_{1}}\left(v_{r_{1}-1}, \delta\right) \leq 2$, completing the proof.

On the other hand, suppose that $d_{Y_{1}}\left(v_{0}, v_{r_{1}}\right)=1$. As $X_{r_{1}-1}$ is connected, $\overline{X_{r_{1}} \backslash X_{r_{1}-1}}$ is either a pair of pants (if $X_{r_{1}-1}$ and $\operatorname{int}\left(S_{r_{1}}\right)$ overlap) or a 4-punctured sphere (if $X_{r_{1}-1}$ and $S_{r_{1}}$ are disjoint). By Lemma 3.3 and our previous assumptions, there is a component $Q_{1}$ of $X_{r_{1}} \cap Y_{1}$ such that $\bar{\chi}_{X_{r_{1}}}\left(Q_{1}\right)=1$. If $Q_{1}$ is a full pair of pants, we refer to Case 2, so we may assume that $Q_{1}$ is either an octagon or a rectangular annulus. In addition, $d_{Y_{1}}\left(v_{r_{1}-1}, v_{r_{1}}\right)>0$ implies that there is an arc $\mu \subset \gamma_{r_{1}} \cap Y_{1}$ such that $\pi_{Y_{1}}(\mu) \notin \pi_{Y_{1}}\left(v_{r_{1}}\right)$. In particular, this implies that $Q_{1}$ meets $\gamma_{r_{1}}$. If $\overline{X_{r_{1}} \backslash X_{r_{1}-1}}$ is a pair of pants, then $Q_{1} \cap X_{r_{1}-1}$ is a collection of rectangles joining distinct boundary components of $X_{r_{1}-1}$, or else $X_{r-1} \cap \partial Y_{1}$ contains a wave and we refer to Case 1, In the former case, each arc of $\gamma_{r_{1}} \cap Y_{1}$ is parallel in $Y_{1}$ to an arc of $\left(v_{0} \cap v_{r_{1}}\right) \cap Y_{1}$, and thus $\pi_{Y_{1}}\left(v_{0}\right) \subset \pi_{Y_{1}}\left(v_{r_{1}}\right)$, a contradiction (see Figure 12). It follows that $\overline{X_{r_{1}} \backslash X_{r_{1}-1}}$ is the 4-punctured sphere $S_{r_{1}}$.

If a boundary arc of $Q_{1} \subset R$ meets $q_{1}$ or $q_{2}$, then $q_{1} \cap X_{r_{1}}$ or $q_{2} \cap X_{r_{1}}$ contains an arc, completing the proof of the theorem as described above. Otherwise, boundary arcs of $Q_{1}$ avoid $q_{1}$ and $q_{2}$. Suppose that $\gamma_{r_{1}}^{\prime}$ meets $Q_{1}$ in an arc $\delta$ and let $\delta^{\prime}$ be any arc of $v_{r_{1}-1} \cap Y_{1}$. If $\delta^{\prime} \cap \delta=\emptyset$, then $d_{Y_{1}}\left(\delta^{\prime}, \delta\right) \leq 2$ by Lemma 3.1. On the other hand, if $\delta^{\prime} \subset Q_{1}$ and $Q_{1}$ is a rectangular annulus, Lemma 3.6 asserts that $d_{Y_{1}}\left(\delta^{\prime}, \delta\right) \leq 2$. If $\delta^{\prime} \subset Q_{1}$ and $Q_{1}$ is an octagon, then we note that $v_{r_{1}-1} \cap X_{m}$ and $v_{r_{1}} \cap X_{m}$ are pants decompositions of $X_{m}, \delta^{\prime} \subset v_{r_{1}-1} \cap R, \delta \subset v_{r_{1}} \cap R$, and both $\delta$ and $\delta^{\prime}$ avoid $q_{1}$ and $q_{2}$. In this case we apply Lemma 3.7, which asserts $d_{Y_{1}}\left(\delta^{\prime}, \delta\right) \leq 2$. In any case, $d_{Y_{1}}\left(v_{0}, \delta\right) \leq d_{Y_{1}}\left(v_{r_{1}-1}, \delta\right) \leq 2$, completing the proof.

Thus, we may assume that $\gamma_{r_{1}}^{\prime}$ avoids $Q_{1}$, and since $Q_{1} \subset S_{r_{1}}$ and $\gamma_{r_{1}}^{\prime}$ cuts $S_{r_{1}}$ into two pairs of pants, $Q_{1}$ cannot be an octagon by Lemma 3.5. For the 


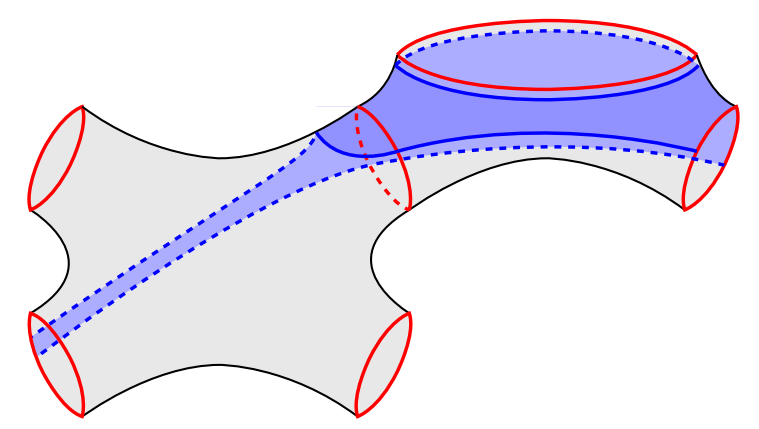

FIGURE 12. The case in which $\overline{X_{r_{1}} \backslash X_{r_{1}-1}}$ is a pair of pants. Here $\gamma_{r_{1}} \cap Y_{1}$ is parallel to an arc of $\partial X_{r_{1}} \cap Y_{1}$.
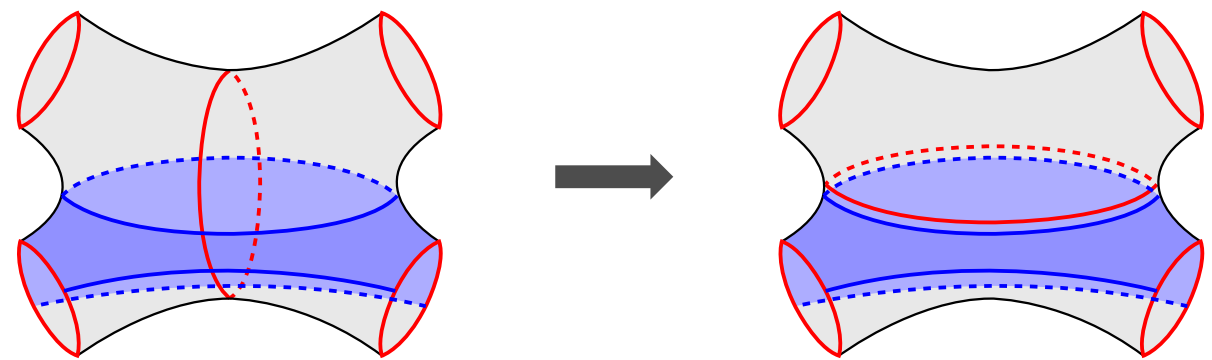

FIGURE 13. Pants move from $v_{r_{1}-1}$ to $v_{r_{1}}$ with support $S_{r_{1}}$.

The curve $\gamma_{r_{1}}^{\prime}$ is isotopic to the curve component $q$ of $\partial Q_{1}$.

remainder of the proof, we suppose that $Q_{1}$ is a rectangular annulus and let $q$ denote the boundary curve of $Q_{1}$. As $\gamma_{r_{1}}^{\prime}$ avoids $Q_{1}$, it must be isotopic to $q$ by Lemma 3.5. In addition, $q \subset \operatorname{int}\left(X_{r_{1}}\right)$ implies $q \subset \partial Y_{1}$ and thus $q \subset \partial R$. See Figure 13. Suppose without loss of generality that $q=q_{1}$. Since $Q_{1}$ is a rectangular annulus, there is an arc component, call it $\beta$, of $\partial Q_{1} \subset v_{r_{1}} \cap Y_{1}$ which is not isotopic to $\alpha$. Note that $\beta$ is a seam in $Y_{1}$ that separates $q_{1}$ from $q_{2}$ in $R$. See Figure 14

Observe that $\beta \subset\left(\partial X_{r_{1}} \cap Y_{1}\right) \subset\left(v_{0} \cap v_{r_{1}}\right) \cap Y_{1}$, so by Lemma3.1 $d_{Y_{1}}\left(v_{0}, \beta\right) \leq$ 2. If $[\beta] \in\left[v_{m} \cap Y_{1}\right]$, then there is an arc $\delta \subset v_{m} \cap R$ isotopic to $\beta$, and $d_{Y_{1}}\left(v_{0}, \delta\right) \leq 2$, completing the proof. Therefore, we suppose that $[\beta] \notin$ $\left[v_{m} \cap Y_{1}\right]$. Let $l$ denote the smallest index such that $[\beta] \notin\left[v_{l} \cap Y_{1}\right]$, let $X_{l-1}^{\prime}$ be the component of $X_{l-1}$ containing $Q_{1}$, and let $X_{l}^{\prime}$ be the component of $X_{l}$ containing $X_{l-1}^{\prime}$. Since $\beta \subset v_{r_{1}} \cap Y_{1}$, we have $r_{1}<l$, and 
by previous assumptions, $\bar{\chi}_{X_{l}^{\prime}}\left(Y_{1}\right)=1$ or 2 (since its value is an integer). We have assumed $[\beta] \in\left[v_{l-1} \cap Y_{1}\right]$ but $[\beta] \notin\left[v_{l} \cap Y_{1}\right]$, which implies that $[\beta] \in\left[\gamma_{l} \cap Y_{1}\right]$. By Lemma 3.1, we have that for any arc $\delta^{\prime}$ of $v_{0} \cap Y_{1}$, either $d_{Y_{1}}\left(\delta^{\prime}, \alpha\right) \leq 1$ or $d_{Y_{1}}\left(\delta^{\prime}, \beta\right) \leq 1$. It follows that $d_{Y_{1}}\left(v_{0}, v_{l-1}\right)=1$. If, in addition, $d_{Y_{1}}\left(v_{0}, v_{l}\right)=1$, then $\bar{\chi}_{X_{l}^{\prime}}\left(Y_{1}\right)=1$ and $X_{l}^{\prime} \cap Y_{1}$ contains a rectangular annulus $D$ such that $Q_{1} \subset D$ and thus $[\beta] \in\left[\partial X_{l}^{\prime} \cap D\right] \subset\left[v_{l} \cap Y_{1}\right]$, a contradiction.

It follows that $d_{Y_{1}}\left(v_{0}, v_{l}\right)=2$, so that $X_{l}^{\prime} \cap Y_{1}$ contains a rectangular pair of pants, which is necessarily contained in $R$. Also, $X_{l}^{\prime} \backslash \eta\left(\gamma_{l}\right)$ has two components, $X_{l-1}^{\prime}$ and another component we will call $S$, where $\bar{\chi}_{S}\left(Y_{1}\right)=1$ and $S \cap Y_{1}$ contains a rectangular annulus $Q^{\prime}$. Let $q^{\prime}$ denote the boundary curve of $Q^{\prime}$, noting that $q^{\prime}$ is isotopic to $q_{2}$.

If $S$ is a pair of pants, $q^{\prime}$ is isotopic to a curve in $\partial X_{l}$. As $Q^{\prime} \subset S_{l}$ we have that $\gamma_{l}^{\prime}$ meets $Q^{\prime}$ by Lemma 3.5, and since $q_{1}$ is isotopic to $\gamma_{r}^{\prime}$, both $\gamma_{l}$ and $\gamma_{l}^{\prime}$ avoid $q_{1}$ and $q_{2}$. Further, $v_{l-1} \cap X_{m}$ and $v_{l} \cap X_{m}$ are pants decompositions of $X_{m}$ which avoid $q_{1}$ and $q_{2}$, so we satisfy the hypotheses of Lemma 3.7 Following the proof of Lemma 3.7 we have that $v_{l-1} \cap R$ contains seams isotopic to $\alpha$ and $\beta$. If $\delta$ is an arc of $\gamma_{l}^{\prime} \cap Q_{1} \subset \gamma_{l}^{\prime} \cap R$, then $\delta$ is disjoint from $\alpha$ and either $\delta$ disjoint from $\beta$, or $\delta$ is one of $\delta_{1}, \delta_{2}$, or $\delta_{3}$, where $\delta_{1}$ is a seam meeting $\beta$ once, $\delta_{2}$ is a wave meeting $\beta$ once, and $\delta_{3}$ is a wave meeting $\beta$ twice, as pictured in Figure 11. Lemma3.7implies that for such $\delta$, we have $d_{Y_{1}}(\alpha, \delta) \leq 1$ unless $\delta=\delta_{1}$, in which case $d_{Y_{1}}\left(\alpha, \delta_{1}\right)=2$, and for all $\delta, d_{Y_{1}}(\beta, \delta) \leq 2$. Now, let $\delta^{\prime}$ be any arc of $v_{0} \cap Y_{1}$ such that $\left[\delta^{\prime}\right] \neq[\alpha],[\beta]$. As $[\alpha],[\beta] \in\left[v_{0} \cap Y_{1}\right]$, we may regard $\delta^{\prime}$ as an arc in $R$ disjoint from $\alpha$ and $\beta$, so by Lemma 3.1, $d_{Y_{1}}\left(\delta^{\prime}, \alpha\right)=1$. For $\delta \neq \delta_{1}$,

$$
d_{Y_{1}}\left(\delta^{\prime}, \delta\right) \leq d_{Y_{1}}\left(\delta^{\prime}, \alpha\right)+d_{Y_{1}}(\alpha, \delta) \leq 2 .
$$

One the other hand, there is a wave $\varepsilon$ disjoint from both $\delta^{\prime}$ and $\delta_{1}$, so

$$
d_{Y_{1}}\left(\delta^{\prime}, \delta\right) \leq d_{Y_{1}}\left(\delta^{\prime}, \varepsilon\right)+d_{Y_{1}}(\varepsilon, \delta) \leq 2 .
$$

Therefore, $d_{Y_{1}}\left(v_{0}, \delta\right) \leq 2$, completing the proof.

Suppose now that $S$ is not a pair of pants, so that $S$ is a component of $X_{l-1}$ that is not $X_{l-1}^{\prime}$. As such, we may (temporarily) commute edges in $\mathscr{P}(\Sigma)$ so that $S=X_{j}$ for some $j$. Recall that $\bar{\chi}_{S}\left(Y_{1}\right)=1$, and since $S=X_{j}$ is connected, we have that $d_{Y_{1}}\left(v_{0}, v_{j}\right)=1$ and there exists an index $s$ such that $d_{Y_{1}}\left(v_{0}, v_{s}\right)=1$ but $d_{Y_{1}}\left(v_{0}, v_{s-1}\right)=0$. A parallel argument to the one above pertaining to $Q_{1}$ shows that there is a component $Q_{2} \subset Q^{\prime}$ of $X_{S} \cap Y_{1}$ contained in the 4-punctured sphere $S_{S}$ such that $Q_{2}$ is a rectangular annulus whose curve boundary $q_{2}$ is isotopic to the curve $\gamma_{s}^{\prime}$, the unique curve in 


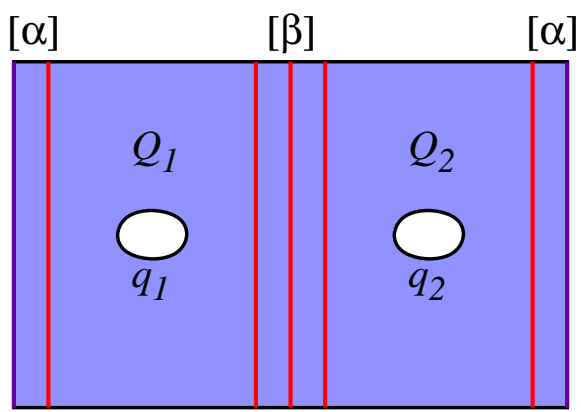

FIGURE 14. The rectangular annulus $R$.

$v_{S} \backslash v_{S-1}$. Now, note that reversing the commutation may result in a reindexing, but the sets of curves $\left\{\gamma_{1}^{\prime}, \ldots, \gamma_{m}^{\prime}\right\}$ and supports $\left\{S_{1}, \ldots, S_{m}\right\}$ are not changed. Thus, we conclude that $s$ corresponds to some index $r_{2}$ after reversing the commutation such that $Q_{2} \subset X_{r_{2}} \cap Y_{1}, Q_{2} \subset S_{r_{2}}$, and the boundary curve $q_{2}$ of $Q_{2}$ is isotopic to $\gamma_{r_{2}}^{\prime}$.

To summarize, we have that $X_{m} \cap Y_{1}=R$, a rectangular annulus, and $R$ is a union of $Q_{1}, Q_{2}$, and (possibly) some rectangles. Further, for $i=1,2$ the arc components of $\operatorname{Fr}_{Y_{1}} Q_{i}$ are isotopic to $\alpha$ and $\beta$, and $\gamma_{r_{i}}^{\prime}$ is isotopic to $q_{i}$, the curve boundary component of $Q_{i}$, which coincides with a curve boundary component of $R$. See Figure 14 for a schematic of $R$. Since $Q_{i} \subset S_{r_{i}}$, it follows that $\Sigma \backslash \eta\left(v_{r_{i}}\right)$ has a pair of pants component $P_{i}$ containing $Q_{i}$. Since $[\beta] \in\left[v_{r_{1}} \cap Y_{1}\right]$ but $[\beta] \notin\left[v_{m} \cap Y_{1}\right]$, there is (at least) one boundary component $\sigma_{i}$ of $P_{i}$ such that $\sigma_{i} \notin v_{m}$. If two boundary components $\sigma_{i}$ and $\sigma_{i}^{\prime}$ of $P_{i}$ are not in $v_{m}$, choose $\sigma_{i}$ so that there is an index $t_{i}$ such that $\sigma_{i} \notin v_{t_{i}}$ but $\sigma_{i}^{\prime} \in v_{t_{i}}$.

There are two final possibilities to consider which will complete the proof of the theorem. First, suppose that $\sigma_{1}=\sigma_{2}$ in $\Sigma$ (see Figure 15). In this case, $Q_{1} \cup Q_{2}$ is contained in the support $S_{t}$ of an elementary move; as such the intersection of $S_{t}$ and $Y_{1}$ contains a rectangular pair of pants, which necessarily meets $\gamma_{t}^{\prime}$ in an arc $\delta$. We note that $v_{t-1} \cap X_{m}$ and $v_{t} \cap X_{m}$ are pants decompositions of $X_{m}$ meeting the hypotheses of Lemma 3.7. Further, as discussed above, since $[\alpha],[\beta] \in v_{0} \cap Y_{1}$, we have that for any arc $\delta^{\prime}$ of $v_{0} \cap Y_{1}$ such that $\left[\delta^{\prime}\right] \neq[\alpha],[\beta]$, either (3) or (4) is satisfied. It follows that $d_{Y_{1}}\left(v_{0}, \delta\right) \leq 2$, completing the proof.

The other possibility is that $\sigma_{1} \neq \sigma_{2}$ (see Figure 16). In this case, we may suppose without loss of generality that $\sigma_{1}$ is replaced in an elementary move before the replacement of $\sigma_{2}$, so that there is an index $t$ such that 


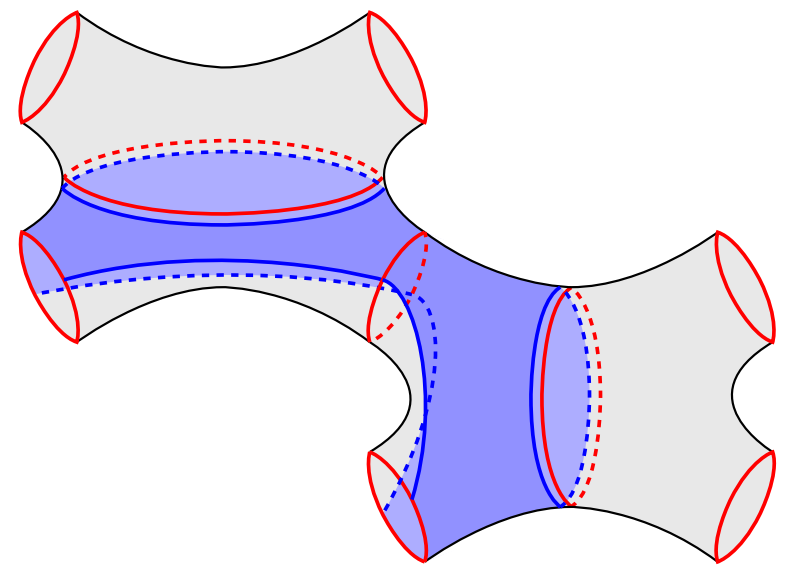

FIGURE 15. A possible embedding of $R$ in $X_{m}$ in the case that $\sigma_{1}=\sigma_{2}$.

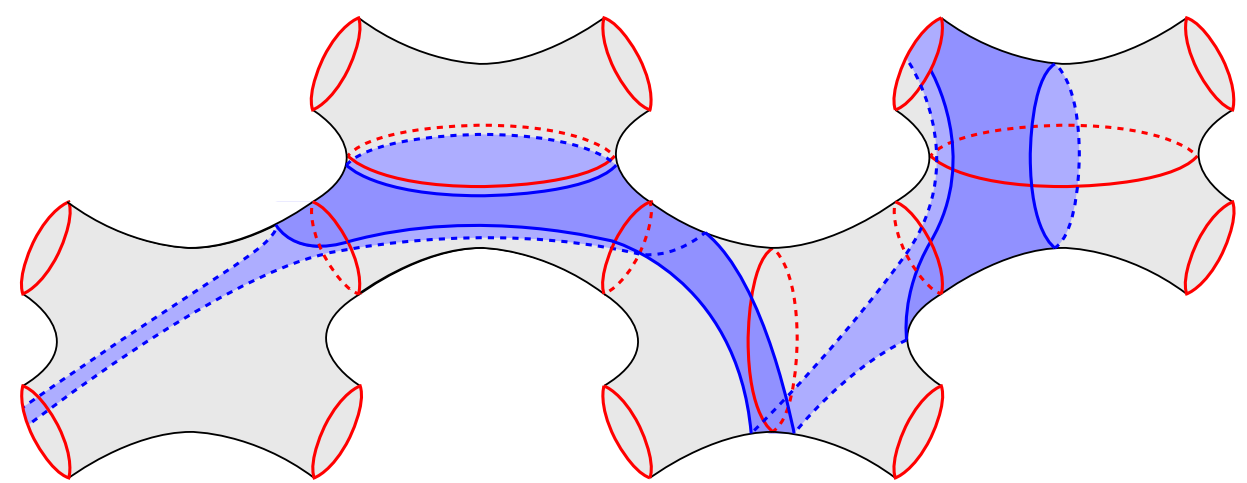

FIGURE 16. A possible embedding of $R$ in $X_{m}$ in the case that $\sigma_{1} \neq \sigma_{2}$. Here we have shown the case in which $\sigma_{1}=\gamma_{t}$ is replaced by an elementary move before $\gamma_{r_{2}}$ (far right) is replaced by $\gamma_{r_{2}}^{\prime}$, isotopic to $q_{2}$.

$\sigma_{1}=\gamma_{t} \notin v_{t}$ and $\sigma_{2} \in v_{t}$. Thus, $\gamma_{t}$ is a boundary component of $P_{1}$ and we have $Q_{1} \subset P_{1} \subset S_{t}$. Further, $q_{1}$ is isotopic to a curve in $\partial S_{t}$, so by Lemma 3.5. $\gamma_{t}^{\prime}$ meets $Q_{1}$ and there is an $\operatorname{arc} \delta \subset \gamma_{t}^{\prime} \cap\left(Y_{1} \backslash \eta\left(Q_{2}\right)\right)$. Let $\delta^{\prime}$ be any arc of $v_{0} \cap Y_{1}$. As $[\alpha],[\beta] \in\left[v_{0} \cap Y_{1}\right]$, we have that either $\delta^{\prime} \subset Q_{2}$ or $\delta \cap Q_{2}=\emptyset$. In the first case, $d_{Y_{1}}\left(\delta^{\prime}, \delta\right) \leq 2$ by Lemma 3.1. Otherwise, $\delta \subset Y_{1} \backslash \eta\left(Q_{2}\right)$, and since $Y_{1} \backslash \eta\left(Q_{2}\right)$ is a rectangular annulus, we may invoke Lemma 3.6 to conclude that $d_{Y_{1}}\left(\delta^{\prime}, \delta\right) \leq 2$. In any case, we have $d_{Y_{1}}\left(v_{0}, \delta\right) \leq 2$, as desired.

This exhausts all possible cases, completing the proof of the lemma. 
Before proceeding, we note that Lemma 4.2 holds for paths of any length, not only those of length $\bar{\chi}(Y)$ or greater.

Proof of the Main Theorem. Let $v_{0}, \ldots, v_{p}$ be a geodesic in $\mathscr{P}(\Sigma)$ such that $v_{0}$ and $v_{p}$ contain $Q$, with $Y=\bigcup Y_{i}$ the complementary subsurface of $Q$. Recall that $d_{Q}\left(v_{0}, v_{p}\right)=d_{Y}\left(v_{0}, v_{p}\right)$, and suppose towards a contradiction that $v_{1}$ does not contain $Q$. Then any curve $\gamma$ in $v_{0} \cap \operatorname{int}(Y)$ is also in $v_{1}$; hence $\pi_{Y}\left(v_{0}\right)=\pi_{Y}\left(v_{1}\right)$. Let $q$ be an index such that

$$
d_{Y}\left(v_{1}, v_{q}\right) \leq q-1
$$

chosen so that $q$ is maximal with respect to all possible commutations of the path $v_{1}, \ldots, v_{p}$. We will prove that $q=p$, which implies that

$$
d\left(v_{0}, v_{p}\right) \leq d_{Q}\left(v_{0}, v_{p}\right)=d_{Y}\left(v_{0}, v_{p}\right)=d_{Y}\left(v_{1}, v_{p}\right)=p-1 .
$$

This will contradict the assumption that $v_{0}, \ldots, v_{p}$ is a geodesic and will complete the proof of the theorem.

Trivially, $q \geq 1$. Let $X_{j}$ denote the support of the path $v_{q}, \ldots, v_{p}$, where $q \leq j \leq p$. By Lemma 4.2, if $X_{p}$ contains a component $Y_{i}$ of $Y$, then after a possible commutation of edges there exists $q^{\prime}$ such that $q<q^{\prime} \leq p$ and $d_{Y}\left(v_{q}, v_{q^{\prime}}\right) \leq q^{\prime}-q$, and thus

$$
d_{Y}\left(v_{1}, v_{q^{\prime}}\right) \leq d_{Y}\left(v_{1}, v_{q}\right)+d_{Y}\left(v_{q}, v_{q^{\prime}}\right) \leq q^{\prime}-1,
$$

contradicting the maximality of $q$. Thus, we may assume that $X_{p}$ does not contain a component $Y_{i}$ of $Y$.

We will show that $v_{p} \cap Y \subset v_{q} \cap Y$. Suppose by way of contradiction that there is an index $i$ and a curve $\gamma^{\prime} \subset v_{p} \cap Y_{i}$ such that $\gamma^{\prime} \notin v_{q}$. Then $\gamma^{\prime} \subset$ $\operatorname{int}\left(X_{p}\right)$. For each curve $\gamma^{\prime \prime} \subset \partial Y_{i}$, either $\gamma^{\prime \prime} \notin v_{q}$, in which case $\gamma^{\prime \prime} \subset \operatorname{int}\left(X_{p}\right)$ or $\gamma^{\prime \prime} \in v_{q}$, in which case $\gamma^{\prime \prime} \subset \partial X_{p}$. In any case, we have $Y_{i} \subset X_{p}$, a contradiction.

Since $v_{p} \cap Y$ contains only curves, $v_{p} \cap Y \subset v_{q} \cap Y$ implies $\pi_{Y}\left(v_{p}\right)=$ $\pi_{Y}\left(v_{q}\right)$ and $d_{Y}\left(v_{q}, v_{p}\right)=0$; thus by the maximality of $q$, we conclude $q=p$. Hence, as noted above, we have that $d\left(v_{0}, v_{p}\right) \leq p-1$, contradicting that our path is a geodesic and completing the proof.

\section{REFERENCES}

[ALPS12] Javier Aramayona, Cyril Lecuire, Hugo Parlier, and Kenneth J Shackleton, Convexity of strata in diagonal pants graphs of surfaces, Publicacions Matemàtiques 57 (2012), no. 1, 219-237.

[APS08] Javier Aramayona, Hugo Parlier, and Kenneth J Shackleton, Totally geodesic subgraphs of the pants complex, Math. Res. Lett 15 (2008), no. 2, 309-320. 
[APS09] Javier Aramayona, Hugo Parlier, and Kenneth Shackleton, Constructing convex planes in the pants complex, Proceedings of the American Mathematical Society 137 (2009), no. 10, 3523-3531.

[Ara10] Javier Aramayona, Simplicial embeddings between pants graphs, Geometriae Dedicata 144 (2010), no. 1, 115-128.

[BF06] Jeffrey Brock and Benson Farb, Curvature and rank of Teichmüller space, American journal of mathematics (2006), 1-22.

[BM07] Jeffrey Brock and Dan Margalit, Weil-petersson isometries via the pants complex, Proceedings of the American Mathematical Society 135 (2007), no. 3, 795-803.

[BM08] Jason A Behrstock and Yair N Minsky, Dimension and rank for mapping class groups, Annals of Mathematics 167 (2008), 1055-1077.

[BMM11] Jeffrey Brock, Howard Masur, and Yair Minsky, Asymptotics of weil-petersson geodesics ii: bounded geometry and unbounded entropy, Geometric and Functional Analysis 21 (2011), no. 4, 820-850.

[Bro01] Jeffrey Brock, Weil-Petersson translation distance and volumes of mapping tori, Comm. Anal. Geom (2001).

[Bro03] , The Weil-Petersson metric and volumes of 3-dimensional hyperbolic convex cores, Journal of the American Mathematical Society 16 (2003), no. 3, 495-535.

[Est13] José L Estévez, Large flats in the pants graph, arXiv preprint arXiv:1306.3170 (2013).

[Ham05] Ursula Hamenstaedt, Geometry of the mapping class groups III: Quasiisometric rigidity, arXiv preprint math/0512429 (2005).

[Joh06] Jesse Johnson, Heegaard splittings and the pants complex, Algebraic \& Geometric Topology 6 (2006), 853-874.

[Mar04] Dan Margalit, Automorphisms of the pants complex, Duke Mathematical Journal 121 (2004), no. 3, 457-480.

[Mas76] Howard Masur, The extension of the Weil-Petersson metric to the boundary of Teichmuller space, Duke Mathematical Journal 43 (1976), no. 3, 623-635.

[MM00] Howard A Masur and Yair N Minsky, Geometry of the complex of curves II: Hierarchical structure, Geometric and Functional Analysis 10 (2000), no. 4, 902-974.

[Wol03] Scott A. Wolpert, Geometry of the Weil-Petersson completion of Teichmüller space, Surveys in Differential Geometry VIII (2003), 357-393.

[Zup13] Alexander Zupan, Bridge and pants complexities of knots, Journal of the London Mathematical Society 87 (2013), no. 1, 43-68.

Department of Mathematics, University of Texas at Austin, 1 UniverSity Station C1200, Austin, TX 78712, U.S.A.

E-mail address: staylor@math.utexas.edu

Department of Mathematics, University of Texas at Austin, 1 UniverSity Station C1200, Austin, TX 78712, U.S.A.

E-mail address: zupan@math.utexas.edu 